\title{
ON THE SINGULARITY OF QUILLEN METRICS
}

\author{
KEN-ICHI YOSHIKAWA
}

\begin{abstract}
Let $\pi: X \rightarrow S$ be a holomorphic map from a compact Kähler manifold $\left(X, g_{X}\right)$ to a compact Riemann surface $S$. Let $\Sigma_{\pi}$ be the critical locus of $\pi$ and let $\Delta=\pi\left(\Sigma_{\pi}\right)$ be the discriminant locus. Let $\left(\xi, h_{\xi}\right)$ be a holomorphic Hermitian vector bundle on $X$. We determine the singularity of the Quillen metric on $\operatorname{det} R \pi_{*} \xi$ near $\Delta$ with respect to $\left.g_{X}\right|_{T X / S}$ and $h_{\xi}$.
\end{abstract}

\section{Introduction}

Let $X$ be a compact Kähler manifold of dimension $n+1$ with Kähler metric $g_{X}$, and let $S$ be a compact Riemann surface. Let $\pi: X \rightarrow S$ be a surjective holomorphic map such that every connected component of $X$ is mapped surjectively to $S$. Let $\Sigma_{\pi}:=\{x \in X ; d \pi(x)=0\}$ be the critical locus of $\pi$. For $t \in S$, set $X_{t}:=\pi^{-1}(t)$. The relative tangent bundle of $\pi: X \rightarrow S$ is the subbundle of $\left.T X\right|_{X \backslash \Sigma_{\pi}}$ defined as $T X / S:=\left.\operatorname{ker} \pi_{*}\right|_{X \backslash \Sigma_{\pi}}$. Set

$$
\Delta:=\pi\left(\Sigma_{\pi}\right), \quad S^{o}:=S \backslash \Delta, \quad X^{o}:=\left.X\right|_{S^{o}}, \quad \pi^{o}:=\left.\pi\right|_{X^{o}} .
$$

Then $\pi^{o}: X^{o} \rightarrow S^{o}$ is a holomorphic family of compact Kähler manifolds. Let $g_{X / S}:=\left.g_{X}\right|_{T X / S}$ be the Hermitian metric on $T X / S$ induced from $g_{X}$.

Let $\xi \rightarrow X$ be a holomorphic vector bundle on $X$ equipped with a Hermitian metric $h_{\xi}$. Let $\lambda(\xi)=\operatorname{det} R \pi_{*} \xi$ be the determinant of the cohomologies of $\xi$. By [5], [14], [15], $\left.\lambda(\xi)\right|_{S^{\circ}}$ is equipped with the Quillen metric $\|\cdot\|_{\lambda(\xi), Q}^{2}$ with respect to the metrics $g_{X / S}$ and $h_{\xi}$.

Let $0 \in \Delta$ be an arbitrary critical value of $\pi$, and let $(\mathcal{U}, t)$ be a coordinate neighborhood of $S$ centered at 0 with $\mathcal{U} \cap \Delta=\{0\}$. Set $\mathcal{U}^{o}:=\mathcal{U} \backslash\{0\}$.

Let $\sigma$ be a nowhere vanishing holomorphic section of $\lambda(\xi)$ on $\mathcal{U}$. Then $\log \|\sigma\|_{\lambda(\xi), Q}^{2}$ is a $C^{\infty}$ function on $\mathcal{U}^{o}$ by [5]. The purpose of this article is to study the behavior of $\log \|\sigma(t)\|_{\lambda(\xi), Q}^{2}$ as $t \rightarrow 0$.

For a holomorphic vector bundle $F$ over a complex manifold with zero-section $Z$, define the projective-space bundle $\mathbb{P}(F)$ as $\mathbb{P}(F):=(F \backslash Z) / \mathbb{C}^{*}$. The dual projective-space bundle $\mathbb{P}(F)^{\vee}$ is defined as $\mathbb{P}(F)^{\vee}:=\mathbb{P}\left(F^{\vee}\right)$, where $F^{\vee}$ is the dual vector bundle of $F$.

Following Bismut [3], we consider the Gauss map $\mu: X \backslash \Sigma_{\pi} \rightarrow \mathbb{P}(T X)^{\vee}$ that assigns $x \in X \backslash \Sigma_{\pi}$ the hyperplane $\operatorname{ker}\left(\pi_{*}\right)_{x} \in \mathbb{P}\left(T_{x} X\right)^{\vee}$. Since $\mu$ extends to a meromorphic map $\mu: X \rightarrow \mathbb{P}(T X)^{\vee}$, there exists a resolution $q:(\widetilde{X}, E) \rightarrow\left(X, \Sigma_{\pi}\right)$ of the indeterminacy of $\mu$ such that $\widetilde{\mu}:=\mu \circ q$ extends to a holomorphic map from $\widetilde{X}$ to $\mathbb{P}(T X)^{\vee}$ and such that $E$ is a normal crossing divisor of $\widetilde{X}$. (For the scheme structure of $E$, see Sect.3.) Let $U$ be the universal hyperplane bundle of rank $n=\operatorname{dim} X / S$ over $\mathbb{P}(T X)^{\vee}$, and let $H:=\mathcal{O}_{\mathbb{P}(T X)^{\vee}}(1)$.

The author is partially supported by the Grants-in-Aid for Scientific Research for Encouragement of Young Scientists (B) 16740030, JSPS. 
After Barlet [1], we define a subspace of $C^{0}(\mathcal{U})$ by

$$
\mathcal{B}(\mathcal{U}):=C^{\infty}(\mathcal{U}) \oplus \bigoplus_{r \in \mathbb{Q} \cap(0,1]} \bigoplus_{k=0}^{n}|t|^{2 r}(\log |t|)^{k} \cdot C^{\infty}(\mathcal{U}) .
$$

A function $\varphi(t) \in \mathcal{B}(\mathcal{U})$ has an asymptotic expansion at $0 \in \Delta$, i.e., there exist $r_{1}, \ldots, r_{m} \in \mathbb{Q} \cap(0,1]$ and $f_{0}, f_{l, k} \in C^{\infty}(\mathcal{U}), l=1, \ldots, m, k=0, \ldots, n$, such that

$$
\varphi(t)=f_{0}(t)+\sum_{l=1}^{m} \sum_{k=0}^{n}|t|^{2 r_{l}}(\log |t|)^{k} f_{l, k}(t) .
$$

In what follows, if $f(t), g(t) \in C^{\infty}\left(\mathcal{U}^{o}\right)$ satisfies $f(t)-g(t) \in \mathcal{B}(\mathcal{U})$, we write

$$
f \equiv_{\mathcal{B}} g \text {. }
$$

For a complex vector bundle $F$ over a complex manifold, $c_{i}(F), \operatorname{Td}(F)$, and $\operatorname{ch}(F)$ denote the $i$-th Chern class, the Todd genus, and the Chern character of $F$, respectively.

We can state the main result of this article, which generalizes $[3, \S 5]$ and [16]:

Theorem 1.1. The following identity holds:

$$
\log \|\sigma\|_{Q, \lambda(\xi)}^{2} \equiv_{\mathcal{B}}\left(\int_{E \cap q^{-1}\left(X_{0}\right)} \tilde{\mu}^{*}\left\{\operatorname{Td}(U) \frac{\operatorname{Td}(H)-1}{c_{1}(H)}\right\} q^{*} \operatorname{ch}(\xi)\right) \log |t|^{2} .
$$

By Theorem 1.1, $\|\cdot\|_{Q, \lambda(\xi)}^{2}$ extends to a singular Hermitian metric on $\lambda(\xi)$. Let $\pi_{*}$ denote the integration along the fibers of $\pi$. As a consequence of Theorem 1.1 and the curvature formula for Quillen metrics [5], we get the following:

Corollary 1.2. The $(1,1)$-form $\pi_{*}\left(\operatorname{Td}\left(T X / S, g_{X / S}\right) \operatorname{ch}\left(\xi, h_{\xi}\right)\right)^{(1,1)}$ lies in $L_{\text {loc }}^{p}(S)$ for some $p>1$, and the curvature current of $\left(\lambda(\xi),\|\cdot\|_{Q, \lambda(\xi)}\right)$ is given by the following formula on $\mathcal{U}$ :

$$
\begin{aligned}
c_{1}\left(\lambda(\xi),\|\cdot\|_{Q, \lambda(\xi)}\right)= & \pi_{*}\left(\operatorname{Td}\left(T X / S, g_{X / S}\right) \operatorname{ch}\left(\xi, h_{\xi}\right)\right)^{(1,1)} \\
& -\left(\int_{E \cap q^{-1}\left(X_{0}\right)} \widetilde{\mu}^{*}\left\{\operatorname{Td}(U) \frac{\operatorname{Td}(H)-1}{c_{1}(H)}\right\} q^{*} \operatorname{ch}(\xi)\right) \delta_{0},
\end{aligned}
$$

where $\delta_{0}$ denotes the Dirac $\delta$-current supported at 0 .

The proof of Theorem 1.1 is quite similar to that of Bismut in $[3, \S 5]$, and we just follow his argument. There are essentially no new ideas except a systematic use of the Gauss maps for the family $\pi: X \rightarrow S$; in fact, the Gauss maps were already used by Bismut in [3].

The existence of an asymptotic expansion of the Quillen norm $\log \|\sigma\|_{Q, \lambda(\xi)}^{2}$ was first shown by Bismut-Bost[4, Sect.13.(b)] when $\pi: X \rightarrow S$ is a family of curves and by the author [16] when $\Sigma_{\pi}$ is isolated. In [9], Theorem 1.1 shall play an crucial role in the study of analytic torsion of Calabi-Yau threefolds.

Let $\mathbf{s}_{\Delta}$ be a section of $\mathcal{O}_{S}(\Delta)$ defining the reduced divisor $\Delta$. Let $\|\cdot\|$ be a $C^{\infty}$ Hermitian metric on $\mathcal{O}_{S}(\Delta)$. By Theorem 1.1,

$$
\log \|\sigma(t)\|_{Q, \lambda(\xi)}^{2}-\left(\int_{E \cap q^{-1}\left(X_{0}\right)} \tilde{\mu}^{*}\left\{\operatorname{Td}(U) \frac{\operatorname{Td}(H)-1}{c_{1}(H)}\right\} q^{*} \operatorname{ch}(\xi)\right) \log \left\|\mathbf{s}_{\Delta}(t)\right\|^{2}
$$

has a finite limit as $t \rightarrow 0$. In Section 6 , we shall compute this limit in terms of various secondary objects, which extends some results in $[3, \S 5]$. 
This article is organized as follows. In Sections 2 and 3, we explain the Gauss maps associated to the family $\pi: X \rightarrow S$ and their resolutions. In Sections 5 and 6 , we prove the main theorem. In Sections 7 and 8 , we verify the compatibility of Theorem 1.1 with the corresponding earlier results of Bismut [3] and the author [16]. In Sections 4 and 9, we prove some technical results. The problem treated in Section 9 seems to be related with the regularity problem of the star products of Green currents [8].

For a complex manifold, we set $d^{c}=\frac{1}{4 \pi i}(\partial-\bar{\partial})$. Hence $d d^{c}=\frac{1}{2 \pi i} \bar{\partial} \partial$. We keep the notation in Sect. 1 throughout this article.

\section{The Gauss maps}

Let $\Omega_{X}^{1}$ be the holomorphic cotangent bundle of $X$. Let $\Pi: \mathbb{P}\left(\Omega_{X}^{1} \otimes \pi^{*} T S\right) \rightarrow X$ be the projective-space bundle associated with $\Omega_{X}^{1} \otimes \pi^{*} T S$. Since $\operatorname{dim} S=1$, we have $\mathbb{P}\left(\Omega_{X}^{1} \otimes \pi^{*} T S\right)=\mathbb{P}\left(\Omega_{X}^{1}\right)$. Let $\Pi^{\vee}: \mathbb{P}(T X)^{\vee} \rightarrow X$ be the dual projective-space bundle of $\mathbb{P}(T X)$, whose fiber $\mathbb{P}\left(T_{x} X\right)^{\vee}$ is the set of hyperplanes of $T_{x} X$ passing through the zero vector of $T_{x} X$. We have the canonical isomorphisms

$$
\mathbb{P}\left(\Omega_{X}^{1} \otimes \pi^{*} T S\right)=\mathbb{P}\left(\Omega_{X}^{1}\right) \cong \mathbb{P}(T X)^{\vee} .
$$

Let $x \in X \backslash \Sigma_{\pi}$. Let $t$ be a holomorphic local coordinate of $S$ near $\pi(x) \in S$. We define the Gauss maps $\nu: X \backslash \Sigma_{\pi} \rightarrow \mathbb{P}\left(\Omega_{X}^{1} \otimes \pi^{*} T S\right)$ and $\mu: X \backslash \Sigma_{\pi} \rightarrow \mathbb{P}(T X)^{\vee}$ by

$$
\nu(x):=\left[d \pi_{x}\right]=\left[\sum_{i=0}^{n} \frac{\partial(t \circ \pi)}{\partial z_{i}}(x) d z_{i} \otimes \frac{\partial}{\partial t}\right], \quad \mu(x):=\left[T_{x} X_{\pi(x)}\right] .
$$

Under the canonical isomorphism $\mathbb{P}\left(\Omega_{X}^{1} \otimes \pi^{*} T S\right) \cong \mathbb{P}(T X)^{\vee}$, one has

$$
\nu=\mu \text {. }
$$

Let

$$
L:=\mathcal{O}_{\mathbb{P}\left(\Omega_{X}^{1} \otimes \pi^{*} T S\right)}(-1) \subset \Pi^{*}\left(\Omega_{X}^{1} \otimes \pi^{*} T S\right)
$$

be the tautological line bundle over $\mathbb{P}\left(\Omega_{X}^{1} \otimes \pi^{*} T S\right)$, and set

$$
Q:=\Pi^{*}\left(\Omega_{X}^{1} \otimes \pi^{*} T S\right) / L \text {. }
$$

We have the exact sequence of holomorphic vector bundles on $\mathbb{P}\left(\Omega_{X}^{1} \otimes \pi^{*} T S\right)$ :

$$
\mathcal{S}: 0 \longrightarrow L \longrightarrow \Pi^{*}\left(\Omega_{X}^{1} \otimes \pi^{*} T S\right) \longrightarrow Q \longrightarrow 0 .
$$

Let $H=\mathcal{O}_{\mathbb{P}\left(T_{X}\right)^{\vee}}(1)$, and let $U$ be the universal hyperplane bundle of $\left(\Pi^{\vee}\right)^{*} T X$. Then the dual of $\mathcal{S}$ is given by

$$
\mathcal{S}^{\vee}: 0 \longrightarrow U \longrightarrow\left(\Pi^{\vee}\right)^{*} T X \longrightarrow H \longrightarrow 0 .
$$

Since $T_{x} X_{\pi(x)}=\left\{v \in T_{x} X ; d \pi_{x}(v)=0\right\}$, we have on $X \backslash \Sigma_{\pi}$

$$
T X / S=\mu^{*} U \text {. }
$$

Let $g_{U}$ be the Hermitian metric on $U$ induced from $\left(\Pi^{\vee}\right)^{*} g_{X}$, and let $g_{H}$ be the Hermitian metric on $H$ induced from $\left(\Pi^{\vee}\right)^{*} g_{X}$ by the $C^{\infty}$-isomorphism $H \cong U^{\perp}$. On $X \backslash \Sigma_{\pi}$, we have

$$
\left(T X / S, g_{\mathcal{X} / S}\right)=\mu^{*}\left(U, g_{U}\right)
$$

Let $g_{S}$ be a Hermitian metric on $S$. Let $g_{\Omega_{X}^{1}}$ be the Hermitian metric on $\Omega_{X}^{1}$ induced from $g_{X}$. Let $g_{L}$ be the Hermitian metric on $L$ induced from the metric $\Pi^{*}\left(g_{\Omega_{X}^{1}} \otimes \pi^{*} g_{S}\right)$ by the inclusion $L \subset \Pi^{*}\left(\Omega_{X}^{1} \otimes \pi^{*} T S\right)$. Let $g_{Q}$ be the Hermitian metric on $Q$ induced from $\Pi^{*}\left(g_{\Omega_{X}^{1}} \otimes \pi^{*} g_{S}\right)$ by the $C^{\infty}$-isomorphism $Q \cong L^{\perp}$. 
Let $c_{1}\left(L, g_{L}\right)$ be the Chern form of $\left(L, g_{L}\right)$. Since $d \pi$ is a nowhere vanishing holomorphic section of $\left.\nu^{*} L\right|_{X \backslash \Sigma_{\pi}}$, we get the following equation on $X \backslash \Sigma_{\pi}$

$$
-d d^{c} \log \|d \pi\|^{2}=\nu^{*} c_{1}\left(L, g_{L}\right) \text {. }
$$

\section{Resolution of the Gauss maps}

Since $\Sigma_{\pi}$ is a proper analytic subset of $X$, the maps $\nu: X \backslash \Sigma_{\pi} \rightarrow \mathbb{P}\left(\Omega_{X}^{1} \otimes \pi^{*} T S\right)$ and $\mu: X \backslash \Sigma_{\pi} \rightarrow \mathbb{P}(T X)^{\vee}$ extend to meromorphic maps $\nu: X \rightarrow \mathbb{P}\left(\Omega_{X}^{1} \otimes \pi^{*} T S\right)$ and $\mu: X \rightarrow \mathbb{P}(T X)^{\vee}$ by [13, Th. 4.5.3]. By Hironaka, there exists a compact Kähler manifold $\widetilde{X}$, a normal crossing divisor $E \subset \widetilde{X}$, a birational holomorphic map $q: \widetilde{X} \rightarrow X$, and holomorphic maps $\widetilde{\nu}: \widetilde{X} \rightarrow \mathbb{P}\left(\Omega_{X}^{1} \otimes \pi^{*} T S\right)$ and $\widetilde{\mu}: \widetilde{X} \rightarrow \mathbb{P}(T X)^{\vee}$ satisfying the following conditions:

(i) $\left.q\right|_{\widetilde{X} \backslash q^{-1}\left(\Sigma_{\pi}\right)}: \widetilde{X} \backslash q^{-1}\left(\Sigma_{\pi}\right) \rightarrow X \backslash \Sigma_{\pi}$ is an isomorphism;

(ii) $q^{-1}\left(\Sigma_{\pi}\right)=E$;

(iii) $(\pi \circ q)^{-1}(b)$ is a normal crossing divisor of $\widetilde{X}$ for all $b \in \Delta$;

(iv) $\widetilde{\nu}=\nu \circ q$ and $\widetilde{\mu}=\mu \circ q$ on $\widetilde{X} \backslash E$.

Then $\widetilde{\nu}=\widetilde{\mu}$ under the canonical isomorphism $\mathbb{P}\left(\Omega_{X}^{1} \otimes \pi^{*} T S\right) \cong \mathbb{P}(T X)^{\vee}$. We set

$$
\widetilde{\pi}:=\pi \circ q
$$

and $\widetilde{X}_{s}:=\widetilde{\pi}^{-1}(s)$ for $s \in S$. Similarly, we set $E_{b}:=E \cap \widetilde{X}_{b}$ for $b \in \Delta$. Sinec $E=q^{-1}\left(\Sigma_{\pi}\right) \subset \widetilde{\pi}^{-1}(\Delta)$, we have $E=\amalg_{b \in \Delta} E_{b}$.

Let $\mathcal{I}_{\Sigma_{\pi}}$ be the ideal sheaf of $\Sigma_{\pi}$. For every $p \in \Sigma_{\pi}$, the sheaf $\mathcal{I}_{\Sigma_{\pi}}$ has the following expression on a neighborhood of $p$ :

$$
\mathcal{I}_{\Sigma_{\pi}}=\mathcal{O}_{X}\left(\frac{\partial(t \circ \pi)}{\partial z_{0}}(z), \cdots, \frac{\partial(t \circ \pi)}{\partial z_{n}}(z)\right) .
$$

Define the ideal sheaf $\mathcal{I}_{E}$ of $E$ as

$$
\mathcal{I}_{E}=q^{-1} \mathcal{I}_{\Sigma_{\pi}}
$$

Denote by $\delta_{E}$ the $(1,1)$-current on $\widetilde{X}$ defined as the integration over $E$, i.e., $\delta_{E}(\psi):=\left.\int_{E} \psi\right|_{E}$ for all $C^{\infty}(n, n)$-form on $\widetilde{X}$. Since $\widetilde{\nu}^{*} L=q^{*} \nu^{*} L, q^{*} d \pi$ extends to a holomorphic section of $\widetilde{\nu}^{*} L$ with zero divisor $E$ by the definition of the ideal sheaf $\mathcal{I}_{E}$. By the Poincaré-Lelong formula, the following identity of currents on $\widetilde{X}$ holds

$$
-d d^{c}\left(q^{*} \log \|d \pi\|^{2}\right)=\widetilde{\nu}^{*} c_{1}\left(L, g_{L}\right)-\delta_{E}
$$

\section{Regularity of the direct image of differential forms}

Recall that $(\mathcal{U}, t)$ is a coordinate neighborhood of $S$ centered at the critical value $0 \in \Delta$. Set $D:=\{(s, t) \in S \times \mathcal{U} ; s=t\}$. Then $D$ is a divisor of $S \times \mathcal{U}$. Let $[D]$ be the line bundle on $S \times \mathcal{U}$ defined by the divisor $D$. Let $\mathbf{s}_{D}$ be a section of $[D]$ with zero divisor $D$. Let $B \subset S$ be a finite subset with $0 \in B$. By shrinking $\mathcal{U}$ if necessary, we may assume that $\mathcal{U} \cap B=\{0\}$. Let $\|\cdot\|_{D}$ be a $C^{\infty}$ Hermitian metric on $[D]$ such that

$$
\left\|\mathbf{s}_{D}(b, t)\right\|_{D}=1, \quad \forall(b, t) \in(B \backslash\{0\}) \times \mathcal{U} .
$$

We set $\mathbf{s}_{t}:=\left.\mathbf{s}_{D}\right|_{S \times\{t\}}$ and $\|\cdot\|_{t}:=\left.\|\cdot\|_{D}\right|_{S \times\{t\}}$ for $t \in \mathcal{U}$. Then $\operatorname{div}\left(\mathbf{s}_{t}\right)=\{t\}$ and $\left\|\mathbf{s}_{t}\right\|_{t}^{2} \in C^{\infty}(S \times \mathcal{U})$.

Let $V$ be a compact connected complex manifold with $\operatorname{dim} V=n+1$. Let $f: V \rightarrow S$ be a proper surjective holomorphic map. We set $V_{t}:=f^{-1}(t)$ for $t \in S$. 
Let $\bar{F}:=\left(F,\|\cdot\|_{F}\right)$ be a holomorphic Hermitian line bundle on $V$, and let $\alpha$ be a holomorphic section of $F$ with

$$
\operatorname{div}(\alpha) \subset \sum_{b \in B} V_{b}
$$

Denote by $f_{*}$ the integration along the fibers of $f$. In Section 4 , we assume that $\varphi$ is a $\partial$-closed and $\bar{\partial}$-closed $C^{\infty}(n, n)$-form on $V$.

Lemma 4.1. There exists a Hörder continuous function $\eta$ on $\mathcal{U}$ such that

$$
f_{*}\left\{\left(\log \|\alpha\|_{F}^{2}\right) \varphi\right\}^{(0,0)}-\left(\int_{\operatorname{div}(\alpha) \cap V_{0}} \varphi\right) \log \left\|\mathbf{s}_{0}\right\|_{0}^{2}=\eta .
$$

Proof. Since $\log \|\alpha\|_{F}^{2} \varphi$ is a locally integrable differential form on $V$, we have $f_{*}\left\{\left(\log \|\alpha\|^{2}\right) \varphi\right\}^{(0,0)} \in L_{\text {loc }}^{1}(S) \cap C^{\infty}\left(S^{o}\right)$. Since $d d^{c}$ commutes with $f_{*}$ and since $\varphi$ is $d$ and $d^{c}$-closed, we get the following equation of currents on $\mathcal{U}$ :

$$
\begin{aligned}
d d^{c} f_{*}\left\{\left(\log \|\alpha\|_{F}^{2}\right) \varphi\right\}^{(0,0)} & =\left[f_{*}\left\{d d^{c}\left(\left(\log \|\alpha\|_{F}^{2}\right) \wedge \varphi\right)\right\}\right]^{(1,1)} \\
& =-\left[f_{*}\left\{\left(c_{1}(\bar{F})-\delta_{\operatorname{div}(\alpha)}\right) \wedge \varphi\right\}\right]^{(1,1)} \\
& =\left(\int_{\operatorname{div}(\alpha) \cap V_{0}} \varphi\right) \delta_{0}-\left[f_{*}\left\{c_{1}(\bar{F}) \wedge \varphi\right\}\right]^{(1,1)} .
\end{aligned}
$$

By Lemma 9.2 below, there exists $\psi \in \mathcal{B}(\mathcal{U})$ such that

$$
\left[f_{*}\left\{c_{1}(\bar{F}) \wedge \varphi\right\}\right]^{(1,1)}(t)=\psi(t) \frac{d t \wedge d \bar{t}}{|t|^{2}}, \quad \psi(0)=0 .
$$

Since $\psi(0)=0$, there exists $\nu \in \mathbb{Q} \cap(0,1]$ such that $\psi(t) \in \sum_{k \leq n}|t|^{2 \nu}(\log |t|)^{k} \cdot \mathcal{B}(\mathcal{U})$. Hence $|t|^{-2} \psi(t) \in L_{\mathrm{loc}}^{p}(\mathcal{U})$ for some $p>1$. By the ellipticity of the Laplacian and the Sobolev embedding theorem, there exists a Hölder continuous function $\chi$ on $\mathcal{U}$ satisfying the following equation of currents on $\mathcal{U}$

$$
\left[f_{*}\left\{c_{1}(\bar{F}) \wedge \varphi\right\}\right]^{(1,1)}=d d^{c} \chi .
$$

This, together with (4.2) and the equation of currents $d d^{c} \log |t|^{2}=\delta_{0}$ on $\mathcal{U}$, implies the assertion, because $\log \left\|\mathbf{s}_{0}\right\|_{0}^{2}-\log |t|^{2} \in C^{\infty}(\mathcal{U})$.

Lemma 4.2. The following identity holds for all $t \in \mathcal{U}^{o}$ :

$$
\begin{aligned}
\int_{V_{t}}\left(\log \|\alpha\|_{F}^{2}\right) \varphi= & \left(\int_{\operatorname{div}(\alpha) \cap V_{0}} \varphi\right) \log \left\|\mathbf{s}_{t}(0)\right\|_{t}^{2}-\int_{V}\left(f^{*} \log \left\|\mathbf{s}_{t}\right\|_{t}^{2}\right) c_{1}(\bar{F}) \wedge \varphi \\
& +\int_{V}\left(\log \|\alpha\|_{F}^{2}\right) f^{*} c_{1}\left([t],\|\cdot\|_{t}\right) \wedge \varphi .
\end{aligned}
$$

Proof. Since $V_{t} \cap \operatorname{div}(\alpha)=\emptyset$ for $t \in \mathcal{U}^{o}, V_{t}$ meets $\operatorname{div}(\alpha)$ properly. Since $\varphi$ is $\partial$ and $\bar{\partial}$-closed, we deduce from [11, Th.2.2.2] the following identity by setting $X=W=V, Y=V_{t}, Z=\operatorname{div}(\alpha)$, and $g_{Y}=-f^{*} \log \left\|\mathbf{s}_{t}\right\|_{t}^{2}, g_{Z}=-\log \|\alpha\|_{F}^{2}$ in [11, 
Sect. 2.2.2]:

$$
\begin{aligned}
\int_{V_{t}}\left(\log \|\alpha\|_{F}^{2}\right) \varphi= & \sum_{b \in B}\left(\int_{\operatorname{div}(\alpha) \cap V_{b}} \varphi\right) \log \left\|\mathbf{s}_{t}(b)\right\|_{t}^{2}-\int_{V}\left(f^{*} \log \left\|\mathbf{s}_{t}\right\|_{t}^{2}\right) c_{1}(\bar{F}) \wedge \varphi \\
& +\int_{V}\left(\log \|\alpha\|_{F}^{2}\right) f^{*} c_{1}\left([t],\|\cdot\|_{t}\right) \wedge \varphi
\end{aligned}
$$

where we used the assumption $\operatorname{div}(\alpha) \subset \sum_{b \in B} V_{b}$. (See also [15, p.59, 1.3-1.7].) Since $\left\|\mathbf{s}_{t}(b)\right\|_{t}=1$ for $(b, t) \in(B \backslash\{0\}) \times \mathcal{U}$ by (4.1), the result follows from (4.3).

Lemma 4.3. The following identity holds

$$
\begin{aligned}
& \lim _{t \rightarrow 0}\left\{\int_{V_{t}}\left(\log \|\alpha\|_{F}^{2}\right) \varphi-\left(\int_{\operatorname{div}(\alpha) \cap V_{0}} \varphi\right) \log \left\|\mathbf{s}_{0}(t)\right\|_{0}^{2}\right\}= \\
& \int_{V}\left(\log \|\alpha\|_{F}^{2}\right) f^{*} c_{1}\left([0],\|\cdot\|_{0}\right) \wedge \varphi-\int_{V}\left(f^{*} \log \left\|\mathbf{s}_{0}\right\|_{0}^{2}\right) c_{1}(\bar{F}) \wedge \varphi .
\end{aligned}
$$

Proof. By Lemma 4.2, we have

$$
\begin{aligned}
\int_{V_{t}}\left(\log \|\alpha\|_{F}^{2}\right) \varphi= & \left(\int_{\operatorname{div}(\alpha) \cap V_{0}} \varphi\right) \log \left\|\mathbf{s}_{0}(t)\right\|_{0}^{2}-\int_{V}\left(f^{*} \log \left\|\mathbf{s}_{t}\right\|_{t}^{2}\right) c_{1}(\bar{F}) \wedge \varphi \\
& +\int_{V}\left(\log \|\alpha\|_{F}^{2}\right) f^{*} c_{1}\left([t],\|\cdot\|_{t}\right) \wedge \varphi+\left(\int_{\operatorname{div}(\alpha) \cap V_{0}} \varphi\right) \log \frac{\left\|\mathbf{s}_{t}(0)\right\|_{t}^{2}}{\left\|\mathbf{s}_{0}(t)\right\|_{0}^{2}}
\end{aligned}
$$

Since $\lim _{s \rightarrow 0} \log \left(\left\|\mathbf{s}_{t}(0)\right\|_{t}^{2} /\left\|\mathbf{s}_{0}(t)\right\|_{0}^{2}\right)=0$, the assertion follows from (4.4).

Lemma 4.4. The following identity of functions on $\mathcal{U}^{o}$ hold:

$$
f_{*}\left\{\left(\log \|\alpha\|_{F}^{2}\right) \varphi\right\}^{(0,0)} \equiv_{\mathcal{B}}\left(\int_{\operatorname{div}(\alpha) \cap V_{0}} \varphi\right) \log \left\|\mathbf{s}_{0}\right\|_{0}^{2} .
$$

Proof. For $t \in \mathcal{U}^{o}$, set

$$
I_{1}(t):=\int_{V}\left(f^{*} \log \left\|\mathbf{s}_{t}\right\|_{t}^{2}\right) c_{1}(\bar{F}) \varphi, \quad I_{2}(t):=\int_{V}\left(\log \|\alpha\|_{F}^{2}\right) f^{*} c_{1}\left([t],\|\cdot\|_{t}\right) \varphi .
$$

By (4.4), it suffices to prove that $I_{1} \in \mathcal{B}(\mathcal{U})$ and $I_{2} \in \mathcal{B}(\mathcal{U})$.

Let $\left\{\left(W_{\lambda}, z_{\lambda}\right)\right\}_{\lambda \in \Lambda}$ be a system of local coordinates on $V$. Since $V$ is compact, we may assume $\# \Lambda<+\infty$. For every $\lambda \in \Lambda$, there exist $F_{\lambda} \in \mathcal{O}\left(W_{\lambda}\right), G_{\lambda} \in \mathcal{O}\left(W_{\lambda}\right)$, $A_{\lambda} \in C^{\infty}\left(W_{\lambda}\right)$, and $B_{\lambda} \in C^{\infty}\left(W_{\lambda} \times \mathcal{U}\right)$ such that

$$
\begin{gathered}
\left.\tilde{\pi}^{*} \log \left\|\mathbf{s}_{t}\right\|_{t}^{2}\right|_{W_{\lambda}}\left(z_{\lambda}\right)=\log \left|F_{\lambda}\left(z_{\lambda}\right)-t\right|^{2}+B_{\lambda}\left(z_{\lambda}, t\right), \\
\left.\log \|\alpha\|_{F}^{2}\right|_{W_{\lambda}}\left(z_{\lambda}\right)=\log \left|G_{\lambda}\left(z_{\lambda}\right)\right|^{2}+A_{\lambda}\left(z_{\lambda}\right) .
\end{gathered}
$$

Let $\left\{\varrho_{\lambda}\right\}_{\lambda \in \Lambda}$ be a partition of unity of $V$ subject to the covering $\left\{W_{\lambda}\right\}_{\lambda \in \Lambda}$. We set $\chi_{\lambda}:=\varrho_{\lambda} c_{1}(\bar{F}) \varphi$. Then

$$
I_{1}(t)=\sum_{\lambda \in \Lambda} \int_{W_{\lambda}} \log \left|F_{\lambda}\left(z_{\lambda}\right)-t\right|^{2} \cdot \chi_{\lambda}\left(z_{\lambda}\right)+\sum_{\lambda \in \Lambda} \int_{W_{\lambda}} B_{\lambda}\left(z_{\lambda}, t\right) \chi_{\lambda}\left(z_{\lambda}\right) .
$$

Since the first term of the right hand side of $(4.5)$ lies in $\mathcal{B}(\mathcal{U})$ by Theorem 9.1 below, we get $I_{1} \in \mathcal{B}(\mathcal{U})$. 
We set $\theta_{\lambda}:=\varrho_{\lambda} \widetilde{\pi}^{*} c_{1}\left([t],\|\cdot\|_{t}\right) \varphi$. Then $\theta_{\lambda}\left(z_{\lambda}, t\right)$ is a $C^{\infty}(n+1, n+1)$-form on $W_{\lambda} \times \mathcal{U}$. Since

$$
I_{2}(t)=\sum_{\lambda \in \Lambda} \int_{W_{\lambda}} \log \left|G_{\lambda}\left(z_{\lambda}\right)\right|^{2} \cdot \theta_{\lambda}\left(z_{\lambda}, t\right)+\sum_{\lambda \in \Lambda} \int_{W_{\lambda}} A_{\lambda}\left(z_{\lambda}\right) \theta_{\lambda}\left(z_{\lambda}, t\right),
$$

we get $I_{2} \in C^{\infty}(\mathcal{U})$. This completes the proof.

Corollary 4.5. The following identity holds

$$
\begin{aligned}
& \lim _{t \rightarrow 0}\left\{\int_{\widetilde{X}_{t}} q^{*}\left(\log \|d \pi\|^{2}\right) \varphi-\left(\int_{E_{0}} \varphi\right) \log \left\|\mathbf{s}_{0}(t)\right\|_{0}^{2}\right\}= \\
& \int_{\widetilde{X}}\left(q^{*} \log \|d \pi\|^{2}\right) \widetilde{\pi}^{*} c_{1}\left([0],\|\cdot\|_{0}\right) \wedge \varphi-\int_{\widetilde{X}}\left(\widetilde{\pi}^{*} \log \left\|\mathbf{s}_{0}\right\|_{0}^{2}\right) \widetilde{\nu}^{*} c_{1}\left(L, g_{L}\right) \wedge \varphi .
\end{aligned}
$$

Proof. Setting $V=\widetilde{X}, f=\widetilde{\pi}, \bar{F}=\widetilde{\nu}^{*}\left(L, g_{L}\right)$ and $\alpha=q^{*}(d \pi)$ in Lemma 4.3, we get the result.

Corollary 4.6. The following identity of functions on $\mathcal{U}^{\circ}$ hold:

$$
\widetilde{\pi}_{*}\left(q^{*}\left(\log \|d \pi\|^{2}\right) \varphi\right)^{(0,0)} \equiv_{\mathcal{B}}\left(\int_{E_{0}} \varphi\right) \log \left\|\mathbf{s}_{0}\right\|_{0}^{2} .
$$

Proof. Setting $V=\widetilde{X}, f=\widetilde{\pi}, \bar{F}=\widetilde{\nu}^{*}\left(L, g_{L}\right)$ and $\alpha=q^{*}(d \pi)$ in Lemma 4.4, we get the result.

\section{Behavior of the Quillen norm of the Knudsen-Mumford section}

Let $\Gamma \subset X \times S$ be the graph of $\pi$, which is a smooth divisor on $X \times S$. Let $[\Gamma]$ be the holomorphic line bundle on $X \times S$ associated to $\Gamma$. Let $s_{\Gamma} \in H^{0}(X \times S,[\Gamma])$ be the canonical section of $[\Gamma]$, so that $\operatorname{div}\left(s_{\Gamma}\right)=\Gamma$. We identify $X$ with $\Gamma$.

Let $i: \Gamma \hookrightarrow X \times S$ be the inclusion. Let $p_{1}: X \times S \rightarrow X$ and $p_{2}: X \times S \rightarrow S$ be the projections. On $X \times S$, we have the exact sequence of coherent sheaves,

$$
0 \longrightarrow \mathcal{O}_{X \times S}\left([\Gamma]^{-1} \otimes p_{1}^{*} \xi\right) \stackrel{\otimes s_{\Gamma}}{\longrightarrow} \mathcal{O}_{X \times S}\left(p_{1}^{*} \xi\right) \longrightarrow i_{*} \mathcal{O}_{\Gamma}\left(p_{1}^{*} \xi\right) \longrightarrow 0 .
$$

Let $\lambda\left(p_{1}^{*} \xi\right), \lambda\left([\Gamma]^{-1} \otimes p_{1}^{*} \xi\right), \lambda(\xi)$ be the determinants of the direct images $R\left(p_{2}\right)_{*} p_{1}^{*} \xi$, $R\left(p_{2}\right)_{*}\left([\Gamma]^{-1} \otimes p_{1}^{*} \xi\right), R \pi_{*} \xi$, respectively. By definition [5], [12], [15],

$$
\lambda(\xi)=\bigotimes_{q \geq 0}\left(\operatorname{det} R^{q} \pi_{*} \xi\right)^{(-1)^{q}} .
$$

Under the isomorphism $\left.p_{1}^{*} \xi\right|_{\Gamma} \cong \xi$ induced from the identification $p_{1}: \Gamma \rightarrow X$, the holomorphic line bundle on $S$

$$
\lambda:=\lambda\left([\Gamma]^{-1} \otimes p_{1}^{*} \xi\right) \otimes \lambda\left(p_{1}^{*} \xi\right)^{-1} \otimes \lambda(\xi)
$$

carries the canonical nowhere vanishing holomorphic section $\sigma_{K M}$ by [7], [12].

Let $\mathcal{V} \subset \mathcal{U}$ be a relatively compact neighborhood of $0 \in \Delta$, and set $\mathcal{V}^{\circ}:=\mathcal{V} \backslash\{0\}$. On $\pi^{-1}(\mathcal{U})$, we identify $\pi$ (resp. $\left.d \pi\right)$ with $t \circ \pi($ resp. $d(t \circ \pi))$. Hence $\pi \in \mathcal{O}\left(\pi^{-1}(\mathcal{U})\right)$ and $d \pi \in H^{0}\left(\pi^{-1}(\mathcal{U}), \Omega_{X}^{1}\right)$ in what follows.

Let $h_{[\Gamma]}$ be a $C^{\infty}$ Hermitian metric on $[\Gamma]$ with

$$
h_{[\Gamma]}\left(s_{\Gamma}, s_{\Gamma}\right)(w, t)=\left\{\begin{array}{llr}
|\pi(w)-t|^{2} & \text { if } & (w, t) \in \pi^{-1}(\mathcal{V}) \times \mathcal{V}, \\
1 & \text { if } & (w, t) \in\left(X \backslash \pi^{-1}(\mathcal{U})\right) \times \mathcal{V} .
\end{array}\right.
$$


Let $h_{[\Gamma]^{-1}}$ be the metric on $[\Gamma]^{-1}$ induced from $h_{[\Gamma]}$.

Let $\|\cdot\|_{Q, \lambda(\xi)}$ be the Quillen metric on $\lambda(\xi)$ with respect to $g_{X / S}, h_{\xi}$. Let $\|\cdot\|_{Q, \lambda\left([\Gamma]^{-1} \otimes p_{1}^{*} \xi\right)}$ (resp. $\left.\|\cdot\|_{Q, \lambda\left(p_{1}^{*} \xi\right)}\right)$ be the Quillen metric on $\lambda\left([\Gamma]^{-1} \otimes p_{1}^{*} \xi\right)$ (resp. $\left.\lambda\left(p_{1}^{*} \xi\right)\right)$ with respect to $g_{X}, h_{[\Gamma]^{-1}} \otimes h_{\xi}\left(\right.$ resp. $\left.g_{X}, h_{\xi}\right)$. Let $\|\cdot\|_{Q, \lambda}$ be the Quillen metric on $\lambda$ defined as the tensor product of those on $\lambda\left([\Gamma]^{-1} \otimes p_{1}^{*} \xi\right), \lambda\left(p_{1}^{*} \xi\right)^{-1}$, $\lambda(\xi)$.

For a complex manifold $Y, A^{p, q}(Y)$ denotes the vector space of $C^{\infty}(p, q)$-forms on $Y$. We set $\widetilde{A}(Y):=\bigoplus_{p>0} A^{p, p}(Y) / \operatorname{Im} \partial+\operatorname{Im} \bar{\partial}$.

For a Hermitian vector bundle $\left(F, h_{F}\right)$ over $Y, c_{i}\left(F, h_{F}\right), \operatorname{Td}\left(F, h_{F}\right), \operatorname{ch}\left(F, h_{F}\right) \in$ $\bigoplus_{p>0} A^{p, p}(Y)$ denote the $i$-th Chern form, the Todd form, and the Chern character form of $\left(F, h_{F}\right)$ with respect to the holomorphic Hermitian connection, respectively. Let $\mathrm{R}(F)$ denote the R-genus of Gillet-Soule $[7,(0.4)]$, [15, p. 160].

Theorem 5.1. The following identity of functions on $\mathcal{U}^{\circ}$ holds

$$
\log \left\|\sigma_{K M}\right\|_{Q, \lambda}^{2} \equiv_{\mathcal{B}}\left(\int_{E_{0}} \tilde{\mu}^{*}\left\{\operatorname{Td}(U) \frac{\operatorname{Td}(H)-1}{c_{1}(H)}\right\} q^{*} \operatorname{ch}(\xi)\right) \log |t|^{2} .
$$

Proof. We follow Bismut [3, Sect. 5]. (See also [17, Th. 6.3].)

(Step 1) Let $\left[X_{t}\right]$ be the holomorphic line bundle on $X$ associated to the divisor $X_{t}$. Then $\left[X_{t}\right]=\left.[\Gamma]\right|_{X_{t}}$. We define the canonical section $s_{t}$ of $\left[X_{t}\right]$ by $s_{t}:=\left.s_{\Gamma}\right|_{X \times\{t\}} \in$ $H^{0}\left(X,\left[X_{t}\right]\right)$. Then $\operatorname{div}\left(s_{t}\right)=X_{t}$. Let $i_{t}: X_{t} \hookrightarrow X$ be the embedding, and set $\xi_{t}:=\left.\xi\right|_{X_{t}}$. By (5.1), we get the exact sequence of coherent sheaves on $X$,

$$
0 \longrightarrow \mathcal{O}_{X}\left(\left[X_{t}\right]^{-1} \otimes \xi\right) \stackrel{\otimes s_{t}}{\longrightarrow} \mathcal{O}_{X}(\xi) \longrightarrow\left(i_{t}\right)_{*} \mathcal{O}_{X_{t}}(\xi) \longrightarrow 0 .
$$

Let $\lambda\left(\left[X_{t}\right]^{-1} \otimes \xi\right)$ and $\lambda\left(\xi_{t}\right)$ be the determinants of the cohomology groups of $\left[X_{t}\right]^{-1} \otimes \xi$ and $\xi_{t}$, respectively. Then $\lambda_{t}=\lambda\left(\left[X_{t}\right]^{-1} \otimes \xi\right) \otimes \lambda(\xi)^{-1} \otimes \lambda\left(\xi_{t}\right)$.

Set $h_{\left[X_{t}\right]}=\left.h_{[\Gamma]}\right|_{X \times\{t\}}$ for $t \in \mathcal{V}$. Then $h_{\left[X_{t}\right]}$ is a Hermitian metric on $\left[X_{t}\right]$. Let $h_{\left[X_{t}\right]}^{-1}$ be the Hermitian metric on $\left[X_{t}\right]^{-1}$ induced from $h_{\left[X_{t}\right]}$.

Let $N_{t}=N_{X_{t} / X}$ (resp. $\left.N_{t}^{*}=N_{X_{t} / X}^{*}\right)$ be the normal (resp. conormal) bundle of $X_{t}$ in $X$. Then $\left.d \pi\right|_{X_{t}} \in H^{0}\left(X_{t}, N_{t}^{*}\right)$ generates $N_{t}^{*}$ for $t \in \mathcal{U}^{o}$. Let $h_{N_{t}^{*}}$ be the Hermitian metric on $N_{t}^{*}$ defined by

$$
h_{N_{t}^{*}}\left(\left.d \pi\right|_{X_{t}},\left.d \pi\right|_{X_{t}}\right)=1 .
$$

Let $h_{N_{t}}$ be the Hermitian metric on $N_{t}$ induced from $h_{N_{t}^{*}}$. Then we have the identity $c_{1}\left(N_{t}, h_{N_{t}}\right)=0$ for $t \in \mathcal{V}^{o}$.

For $(w, t) \in \pi^{-1}(\mathcal{U}) \times \mathcal{U}$, set

$$
\widetilde{s}_{\Gamma}(w, t)=\frac{s_{\Gamma}(w, t)}{\pi(w)-t} .
$$

Since $\pi(w)-t$ is a holomorphic function on $\pi^{-1}(\mathcal{U}) \times \mathcal{U}$ with divisor $\Gamma, \widetilde{s}_{\Gamma}$ is a

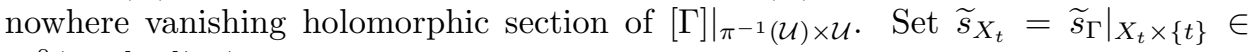
$H^{0}\left(X_{t},\left.\left[X_{t}\right]\right|_{X_{t}}\right)$ and

$$
\left.d s_{t}\right|_{X_{t}}:=d \pi \otimes \widetilde{s}_{X_{t}} \in H^{0}\left(X_{t},\left.N_{t}^{*} \otimes\left[X_{t}\right]\right|_{X_{t}}\right) .
$$

By (5.2), (5.4), the isomorphism

$$
\left.\otimes d s_{t}\right|_{X_{t}}:\left.\left.\left[X_{t}\right]^{-1} \otimes \xi\right|_{X_{t}} \ni v \rightarrow d s_{t}\right|_{X_{t}}(v) \in N_{t}^{*} \otimes \xi_{t}
$$

gives an isometry of holomorphic Hermitian vector bundles

$$
\left.\left(\left[X_{t}\right]^{-1} \otimes \xi, h_{\left[X_{t}\right]^{-1}} \otimes h_{\xi}\right)\right|_{X_{t}} \cong\left(N_{t}^{*} \otimes \xi_{t},\left.h_{N_{t}^{*}} \otimes h_{\xi}\right|_{X_{t}}\right)
$$


for all $t \in \mathcal{V}^{o}$. Hence the metrics $h_{\left[X_{t}\right]^{-1}} \otimes h_{\xi}$ and $h_{\xi}$ verify assumption (A) of Bismut [2, Def.1.5] with respect to $h_{N_{t}}$ and $\left.h_{\xi}\right|_{X_{t}}$.

(Step 2) Associated to the exact sequence of holomorphic vector bundles on $X_{t}$,

$$
\mathcal{E}_{t}:\left.0 \longrightarrow T X_{t} \longrightarrow T X\right|_{X_{t}} \longrightarrow N_{t} \longrightarrow 0,
$$

one can define the Bott-Chern class $\widetilde{\mathrm{Td}}\left(\mathcal{E}_{t} ; g_{X_{t}}, g_{X}, h_{N_{t}}\right) \in \widetilde{A}\left(X_{t}\right)$ by [5, I, f)], [10, I, Sect. 1], [15, Chap.IV, Sect. 3] such that

$$
d d^{c} \widetilde{\operatorname{Td}}\left(\mathcal{E}_{t} ; g_{X_{t}}, g_{X}, h_{N_{t}}\right)=\operatorname{Td}\left(T X_{t}, g_{X_{t}}\right) \operatorname{Td}\left(N_{t}, h_{N_{t}}\right)-\left.\operatorname{Td}\left(T X, g_{X}\right)\right|_{X_{t}} .
$$

Notice that our $\widetilde{\operatorname{Td}}\left(\mathcal{E}_{t} ; g_{X_{t}}, g_{X}, h_{N_{t}}\right)$ and Bismut-Lebeau's $\widetilde{\operatorname{Td}}\left(T X_{t},\left.T X\right|_{X_{t}}, h_{N_{t}}\right)$ are related as follows:

$$
\widetilde{\operatorname{Td}}\left(\mathcal{E}_{t} ; g_{X_{t}}, g_{X}, h_{N_{t}}\right)=-\widetilde{\operatorname{Td}}\left(T X_{t},\left.T X\right|_{X_{t}}, h_{N_{t}}\right) .
$$

Let $Z$ be a general fiber of $\pi: X \rightarrow S$. By applying the embedding formula of Bismut-Lebeau [7, Th. 0.1] (see also [3, Th. 5.6]) to the embedding $i_{t}: X_{t} \hookrightarrow X$ and to the exact sequence (5.3), we get for all $t \in \mathcal{V}^{o}$ :

$$
\begin{aligned}
\log \left\|\sigma_{K M}(t)\right\|_{Q, \lambda}^{2}= & \int_{X \times\{t\}}-\left.\frac{\operatorname{Td}\left(T X, g_{X}\right) \operatorname{ch}\left(\xi, h_{\xi}\right)}{\operatorname{Td}\left([\Gamma], h_{[\Gamma]}\right)} \log h_{[\Gamma]}\left(s_{\Gamma}, s_{\Gamma}\right)\right|_{X \times\{t\}} \\
& -\int_{X_{t}} \frac{\widetilde{T d}\left(\mathcal{E}_{t} ; g_{X_{t}}, g_{X}, h_{N_{t}}\right) \operatorname{ch}\left(\xi, h_{\xi}\right)}{\operatorname{Td}\left(N_{t}, h_{N_{t}}\right)} \\
& -\int_{X} \operatorname{Td}(T X) R(T X) \operatorname{ch}(\xi)+\int_{Z} \operatorname{Td}(T Z) R(T Z) \operatorname{ch}\left(\left.\xi\right|_{Z}\right) .
\end{aligned}
$$

Here we used the explicit formula for the Bott-Chern current $[6$, Rem. 3.5, especially (3.23), Th. 3.15, Th. 3.17] to get the first term of the right hand side of (5.5). Notice that the dual of our $\lambda(\xi)$ was defined as $\lambda(\xi)$ in [7].

By Theorem 9.1 below, the first term of the right hand side of $(5.5)$ lies in $\mathcal{B}(\mathcal{U})$. Substituting $c_{1}\left(N_{t}, h_{N_{t}}\right)=0$ into (5.5), we get

$$
\log \left\|\sigma_{K M}(t)\right\|_{Q, \lambda}^{2} \equiv_{\mathcal{B}} \int_{X_{t}}-\widetilde{\operatorname{Td}}\left(\mathcal{E}_{t} ; g_{X_{t}}, g_{X}, h_{N_{t}}\right) \operatorname{ch}\left(\xi, h_{\xi}\right) .
$$

(Step 3) Let $g_{N_{t}}$ be the Hermitian metric on $N_{t}$ induced from $g_{X}$ by the $C^{\infty}$ isomorphism $N_{t} \cong\left(T X_{t}\right)^{\perp}$. Let $\widetilde{\mathrm{Td}}\left(N_{t} ; h_{N_{t}}, g_{N_{t}}\right) \in \widetilde{A}\left(X_{t}\right)$ be the Bott-Chern class [5, I, e)], [10, Sect. 1.2.4], [15, Chap. IV, Sect. 3] such that

$$
d d^{c} \widetilde{\operatorname{Td}}\left(N_{t} ; h_{N_{t}}, g_{N_{t}}\right)=\operatorname{Td}\left(N_{t}, h_{N_{t}}\right)-\operatorname{Td}\left(N_{t}, g_{N_{t}}\right)
$$

By [10, I, Prop. 1.3.2 and Prop. 1.3.4] (see also Lemma 5.3 below),

$$
\widetilde{\operatorname{Td}}\left(\mathcal{E}_{t} ; g_{X_{t}}, g_{X}, h_{N_{t}}\right)=\widetilde{\operatorname{Td}}\left(\mathcal{E}_{t} ; g_{X_{t}}, g_{X}, g_{N_{t}}\right)+\operatorname{Td}\left(T X_{t}, g_{X_{t}}\right) \widetilde{\operatorname{Td}}\left(N_{t} ; h_{N_{t}}, g_{N_{t}}\right) \text {. }
$$

Since $c_{1}\left(N_{t}, h_{N_{t}}\right)=0$ and $g_{N_{t}}=\|d \pi\|^{-2} h_{N_{t}}$, we deduce from [10, I, Prop. 1.3.1 and (1.2.5.1)] the identity

$$
\begin{aligned}
\widetilde{\operatorname{Td}}\left(N_{t} ; h_{N_{t}}, g_{N_{t}}\right) & =\frac{1-\operatorname{Td}\left(d d^{c} \log \|d \pi\|^{2}\right)}{d d^{c} \log \|d \pi\|^{2}} \log \|d \pi\|^{2} \\
& =\left.\nu^{*}\left\{\frac{1-\operatorname{Td}\left(-c_{1}\left(L, g_{L}\right)\right)}{-c_{1}\left(L, g_{L}\right)}\right\} \log \|d \pi\|^{2}\right|_{X_{t}} .
\end{aligned}
$$


Substituting (5.8) and $\left(T X_{t}, g_{X_{t}}\right)=\left.\mu^{*}\left(U, g_{U}\right)\right|_{X_{t}}$ into (5.7), we get

$$
\begin{aligned}
& \widetilde{\operatorname{Td}}\left(\mathcal{E}_{t} ; g_{X_{t}}, g_{X}, h_{N_{t}}\right)= \\
& \widetilde{\operatorname{Td}}\left(\mathcal{E}_{t} ; g_{X_{t}}, g_{X}, g_{N_{t}}\right)+\left.\mu^{*} \operatorname{Td}\left(U, g_{U}\right) \nu^{*}\left\{\frac{1-\operatorname{Td}\left(-c_{1}\left(L, g_{L}\right)\right)}{-c_{1}\left(L, g_{L}\right)}\right\} \log \|d \pi\|^{2}\right|_{X_{t}} .
\end{aligned}
$$

Since

$$
\mathcal{E}_{t}=\left.\mu^{*} \mathcal{S}^{\vee}\right|_{X_{t}}, \quad g_{X_{t}}=\left.\mu^{*} g_{U}\right|_{X_{t}}, \quad g_{X}=\left.\mu^{*}\left(\Pi^{\vee}\right)^{*} g_{X}\right|_{X_{t}}, \quad g_{N_{t}}=\left.\mu^{*} g_{H}\right|_{X_{t}},
$$

we deduce from $[10$, I, Th. 1.2.2 (ii)] that

$$
\widetilde{\operatorname{Td}}\left(\mathcal{E}_{t} ; g_{X_{t}}, g_{X}, g_{N_{t}}\right)=\left.\mu^{*} \widetilde{\operatorname{Td}}\left(\mathcal{S}^{\vee} ; g_{U},\left(\Pi^{\vee}\right)^{*} g_{X}, g_{H}\right)\right|_{X_{t}} .
$$

Comparing (5.9) and (5.10), we get

$$
\begin{aligned}
\widetilde{\operatorname{Td}}\left(\mathcal{E}_{t} ; g_{X_{t}}, g_{X}, h_{N_{t}}\right)= & \left.\mu^{*} \widetilde{\operatorname{Td}}\left(\mathcal{S}^{\vee} ; g_{U},\left(\Pi^{\vee}\right)^{*} g_{X}, g_{H}\right)\right|_{X_{t}} \\
& +\left.\mu^{*} \operatorname{Td}\left(U, g_{U}\right) \nu^{*}\left\{\frac{1-\operatorname{Td}\left(-c_{1}\left(L, g_{L}\right)\right)}{-c_{1}\left(L, g_{L}\right)}\right\} \log \|d \pi\|^{2}\right|_{X_{t}} .
\end{aligned}
$$

Substituting (5.11) into (5.6), we get

$$
\begin{aligned}
& \log \left\|\sigma_{K M}\right\|_{Q, \lambda}^{2} \\
& \equiv_{\mathcal{B}}-\pi_{*}\left[\mu^{*} \widetilde{\operatorname{Td}}\left(\mathcal{S}^{\vee} ; g_{U},\left(\Pi^{\vee}\right)^{*} g_{X}, g_{H}\right) \operatorname{ch}\left(\xi, h_{\xi}\right)\right]^{(0,0)} \\
& \quad-\pi_{*}\left[\mu^{*} \operatorname{Td}\left(U, g_{U}\right) \nu^{*}\left\{\frac{1-\operatorname{Td}\left(-c_{1}\left(L, g_{L}\right)\right)}{-c_{1}\left(L, g_{L}\right)}\right\} \operatorname{ch}\left(\xi, h_{\xi}\right) \log \|d \pi\|^{2}\right]^{(0,0)} \\
& \equiv_{\mathcal{B}}-\widetilde{\pi}_{*}\left[\widetilde{\mu}^{*} \widetilde{\operatorname{Td}}\left(\mathcal{S}^{\vee} ; g_{U},\left(\Pi^{\vee}\right)^{*} g_{X}, g_{H}\right) q^{*} \operatorname{ch}\left(\xi, h_{\xi}\right)\right]^{(0,0)} \\
& \quad+\widetilde{\pi}_{*}\left[\widetilde{\mu}^{*} \operatorname{Td}\left(U, g_{U}\right) \widetilde{\nu}^{*}\left\{\frac{\operatorname{Td}\left(-c_{1}\left(L, g_{L}\right)\right)-1}{-c_{1}\left(L, g_{L}\right)}\right\} q^{*} \operatorname{ch}\left(\xi, h_{\xi}\right)\left(q^{*} \log \|d \pi\|^{2}\right)\right]^{(0,0)} .
\end{aligned}
$$

Recall that for a $C^{\infty}$ differential form $\varphi$ on $\widetilde{X}$, one has $\widetilde{\pi}_{*}(\varphi)^{(0,0)} \in \mathcal{B}(\mathcal{U})$ by Barlet [1, Th. 4bis]. Since $q^{*} \operatorname{ch}\left(\xi, h_{\xi}\right)$ and

$$
\widetilde{\mu}^{*} \widetilde{\operatorname{Td}}\left(\mathcal{S}^{\vee} ; g_{U},\left(\Pi^{\vee}\right)^{*} g_{X}, g_{H}\right), \quad \widetilde{\mu}^{*} \operatorname{Td}\left(U, g_{U}\right), \quad \widetilde{\nu}^{*}\left\{\frac{\operatorname{Td}\left(-c_{1}\left(L, g_{L}\right)\right)-1}{-c_{1}\left(L, g_{L}\right)}\right\}
$$

are $C^{\infty}$ differential forms on $\widetilde{X}$, we deduce from (5.12), [1, Th. 4bis], and Corollary 4.6 that

$$
\log \left\|\sigma_{K M}\right\|_{Q, \lambda}^{2} \equiv_{\mathcal{B}}\left(\int_{E_{0}} \widetilde{\mu}^{*}\left\{\operatorname{Td}(U) \frac{\operatorname{Td}(H)-1}{c_{1}(H)}\right\} q^{*} \operatorname{ch}(\xi)\right) \log |t|^{2} .
$$

Here we used the identity $c_{1}(H)=-c_{1}(L)+\left(\Pi^{\vee}\right)^{*} \pi^{*} c_{1}(S)$ in $H^{2}\left(\mathbb{P}(T X)^{\vee}, \mathbb{Z}\right)$ and the triviality of the line bundle $\left.\widetilde{\mu}^{*}\left(\Pi^{\vee}\right)^{*} \pi^{*}(T S)\right|_{\widetilde{\pi}^{-1}(\mathcal{U})}$ to get (5.13). This completes the proof of Theorem 5.1. 
For simplicity, we set $\bar{L}:=\left(L, g_{L}\right), \bar{U}:=\left(U, g_{U}\right), \bar{\xi}:=\left(\xi, h_{\xi}\right)$ in what follows.

Let $\widetilde{\operatorname{Td}}\left(\mathcal{S}^{\vee} ; g_{U},\left(\Pi^{\vee}\right)^{*} g_{X}, g_{H}\right)$ be the Bott-Chern secondary class associated with the Todd genus and the exact sequence of holomorphic vector bundles

$$
\mathcal{S}^{\vee}: 0 \rightarrow U \rightarrow\left(\Pi^{\vee}\right)^{*} T X \rightarrow H \rightarrow 0
$$

equipped with the Hermitian metrics $g_{U},\left(\Pi^{\vee}\right)^{*} g_{X}, g_{H}$, such that

$$
d d^{c} \widetilde{\operatorname{Td}}\left(\mathcal{S}^{\vee} ; g_{U},\left(\Pi^{\vee}\right)^{*} g_{X}, g_{H}\right)=\operatorname{Td}\left(U, g_{U}\right) \operatorname{Td}\left(H, g_{H}\right)-\left(\Pi^{\vee}\right)^{*} \operatorname{Td}\left(T X, g_{X}\right) .
$$

Recall that $Z$ is a general fiber of $\pi: X \rightarrow S$.

Theorem 5.2. The following identity holds

$$
\begin{aligned}
& \lim _{t \rightarrow 0}\left[\log \left\|\sigma_{K M}(t)\right\|_{Q, \lambda}^{2}-\left(\int_{E_{0}} \widetilde{\mu}^{*}\left\{\operatorname{Td}(U) \frac{\operatorname{Td}(H)-1}{c_{1}(H)}\right\} q^{*} \operatorname{ch}(\xi)\right) \log \left\|\mathbf{s}_{0}(t)\right\|_{0}^{2}\right]= \\
& -\left.\int_{X \times\{0\}} \frac{\operatorname{Td}\left(T X, g_{X}\right) \operatorname{ch}(\bar{\xi})}{\operatorname{Td}\left([\Gamma], h_{[\Gamma]}\right)} \log \left\|s_{\Gamma}\right\|^{2}\right|_{X \times\{0\}} \\
& -\int_{\widetilde{X}_{0}} \widetilde{\mu}^{*} \widetilde{\operatorname{Td}}\left(\mathcal{S}^{\vee} ; g_{U},\left(\Pi^{\vee}\right)^{*} g_{X}, g_{H}\right) q^{*} \operatorname{ch}(\bar{\xi}) \\
& +\int_{\widetilde{X}}\left(q^{*} \log \|d \pi\|^{2}\right) \widetilde{\pi}^{*} c_{1}\left([0],\|\cdot\|_{0}\right)\left[\widetilde{\mu}^{*} \operatorname{Td}(\bar{U}) \widetilde{\nu}^{*}\left\{\frac{\operatorname{Td}\left(-c_{1}(\bar{L})\right)-1}{-c_{1}(\bar{L})}\right\} q^{*} \operatorname{ch}(\bar{\xi})\right] \\
& -\int_{\widetilde{X}}\left(\widetilde{\pi}^{*} \log \left\|\mathbf{s}_{0}\right\|_{0}^{2}\right) \widetilde{\nu}^{*} c_{1}(\bar{L})\left[\widetilde{\mu}^{*} \operatorname{Td}(\bar{U}) \widetilde{\nu}^{*}\left\{\frac{\operatorname{Td}\left(-c_{1}(\bar{L})-1\right.}{-c_{1}(\bar{L})}\right\} q^{*} \operatorname{ch}(\bar{\xi})\right] \\
& -\int_{X} \operatorname{Td}(T X) \operatorname{R}(T X) \operatorname{ch}(\xi)+\int_{Z} \operatorname{Td}(T Z) \operatorname{R}(T Z) \operatorname{ch}\left(\left.\xi\right|_{Z}\right) .
\end{aligned}
$$

Proof. Define topological constants $C_{0}$ and $C_{1}$ by

$$
\begin{gathered}
C_{0}:=\int_{E_{0}} \tilde{\mu}^{*}\left\{\operatorname{Td}(U) \frac{\operatorname{Td}(H)-1}{c_{1}(H)}\right\} q^{*} \operatorname{ch}(\xi), \\
C_{1}:=-\int_{X} \operatorname{Td}(T X) \operatorname{R}(T X) \operatorname{ch}(\xi)+\int_{Z} \operatorname{Td}(T Z) \operatorname{R}(T Z) \operatorname{ch}\left(\left.\xi\right|_{Z}\right) .
\end{gathered}
$$

Substituting (5.11) and $c_{1}\left(N_{t}, h_{N_{t}}\right)=0$ into (5.5), we get for $t \in \mathcal{U}^{o}$

$$
\begin{aligned}
\log \left\|\sigma_{K M}(t)\right\|_{Q, \lambda}^{2}= & -\left.\int_{X \times\{t\}} \frac{\operatorname{Td}\left(T X, g_{X}\right) \operatorname{ch}(\bar{\xi})}{\operatorname{Td}\left([\Gamma], h_{[\Gamma]}\right)} \log \left\|s_{\Gamma}\right\|^{2}\right|_{X \times\{t\}} \\
& -\left.\int_{X_{t}} \mu^{*} \widetilde{\operatorname{Td}}\left(\mathcal{S}^{\vee} ; g_{U},\left(\Pi^{\vee}\right)^{*} g_{X}, g_{H}\right)\right|_{X_{t}} \operatorname{ch}(\bar{\xi}) \\
& -\int_{X_{t}} \mu^{*} \operatorname{Td}(\bar{U}) \nu^{*}\left\{\frac{1-\operatorname{Td}\left(-c_{1}(\bar{L})\right)}{-c_{1}(\bar{L})}\right\} \operatorname{ch}(\bar{\xi}) \log \|d \pi\|^{2}+C_{1} \\
= & -\left.\int_{X \times\{t\}} \frac{\operatorname{Td}\left(T X, g_{X}\right) \operatorname{ch}(\bar{\xi})}{\operatorname{Td}\left([\Gamma], h_{[\Gamma]}\right)} \log \left\|s_{\Gamma}\right\|^{2}\right|_{X \times\{t\}} \\
& -\left.\int_{\widetilde{X}_{t}} \widetilde{\mu}^{*} \widetilde{\operatorname{Td}}\left(\mathcal{S}^{\vee} ; g_{U},\left(\Pi^{\vee}\right)^{*} g_{X}, g_{H}\right)\right|_{X_{t}} q^{*} \operatorname{ch}(\bar{\xi}) \\
& +\int_{\widetilde{X}_{t}} \widetilde{\mu}^{*} \operatorname{Td}(\bar{U}) \widetilde{\nu}^{*}\left\{\frac{\operatorname{Td}\left(-c_{1}(\bar{L})\right)-1}{-c_{1}(\bar{L})}\right\} q^{*} \operatorname{ch}(\bar{\xi}) q^{*}\left(\log \|d \pi\|^{2}\right)+C_{1},
\end{aligned}
$$


which yields that

$$
\begin{aligned}
& \log \left\|\sigma_{K M}(t)\right\|_{Q, \lambda}^{2}-C_{0} \log \left\|\mathbf{s}_{0}(t)\right\|_{0}^{2}= \\
& -\int_{X \times\{t\}} \frac{\operatorname{Td}\left(T X, g_{X}\right) \operatorname{ch}(\bar{\xi})}{\operatorname{Td}\left([\Gamma], h_{[\Gamma]}\right)} \log \left\|s_{\Gamma}\right\|^{2}-\int_{\widetilde{X}_{t}} \widetilde{\mu}^{*} \widetilde{\operatorname{Td}}\left(\mathcal{S}^{\vee} ; g_{U},\left(\Pi^{\vee}\right)^{*} g_{X}, g_{H}\right) q^{*} \operatorname{ch}(\bar{\xi}) \\
& +\int_{\widetilde{X}_{t}}\left[\widetilde{\mu}^{*} \operatorname{Td}(\bar{U}) \widetilde{\nu}^{*}\left\{\frac{\operatorname{Td}\left(-c_{1}(\bar{L})\right)-1}{-c_{1}(\bar{L})}\right\} q^{*} \operatorname{ch}(\bar{\xi})\right] q^{*}\left(\log \|d \pi\|^{2}\right)-C_{0} \log \left\|\mathbf{s}_{0}(t)\right\|_{0}^{2} \\
& +C_{1} .
\end{aligned}
$$

By Corollary 4.5,

$$
\begin{aligned}
& \int_{\widetilde{X}_{t}}\left[\widetilde{\mu}^{*} \operatorname{Td}(\bar{U}) \widetilde{\nu}^{*}\left\{\frac{\operatorname{Td}\left(-c_{1}(\bar{L})\right)-1}{-c_{1}(\bar{L})}\right\} q^{*} \operatorname{ch}(\bar{\xi})\right] q^{*}\left(\log \|d \pi\|^{2}\right)-C_{0} \log \left\|\mathbf{s}_{0}(t)\right\|_{0}^{2} \\
& =\int_{\widetilde{X}}\left(q^{*} \log \|d \pi\|^{2}\right) \widetilde{\pi}^{*} c_{1}\left([0],\|\cdot\|_{0}\right)\left[\widetilde{\mu}^{*} \operatorname{Td}(\bar{U}) \widetilde{\nu}^{*}\left\{\frac{\operatorname{Td}\left(-c_{1}(\bar{L})\right)-1}{-c_{1}(\bar{L})}\right\} q^{*} \operatorname{ch}(\bar{\xi})\right] \\
& -\int_{\widetilde{X}}\left(\widetilde{\pi}^{*} \log \left\|\mathbf{s}_{0}\right\|_{0}^{2}\right) \widetilde{\nu}^{*} c_{1}(\bar{L})\left[\widetilde{\mu}^{*} \operatorname{Td}(\bar{U}) \widetilde{\nu}^{*}\left\{\frac{\operatorname{Td}\left(-c_{1}(\bar{L})-1\right.}{-c_{1}(\bar{L})}\right\} q^{*} \operatorname{ch}(\bar{\xi})\right]+o(1) .
\end{aligned}
$$

From (5.15) and (5.16), we get

$$
\begin{aligned}
& \lim _{t \rightarrow 0}\left[\log \left\|\sigma_{K M}(t)\right\|_{Q, \lambda}^{2}-C_{0} \log \left\|\mathbf{s}_{0}(t)\right\|_{0}^{2}\right]= \\
& -\left.\int_{X \times\{0\}} \frac{\operatorname{Td}\left(T X, g_{X}\right) \operatorname{ch}(\bar{\xi})}{\operatorname{Td}\left([\Gamma], h_{[\Gamma]}\right)} \log \left\|s_{\Gamma}\right\|^{2}\right|_{X \times\{0\}} \\
& -\int_{\widetilde{X}_{0}} \widetilde{\mu}^{*} \widetilde{\operatorname{Td}}\left(\mathcal{S}^{\vee} ; g_{U},\left(\Pi^{\vee}\right)^{*} g_{X}, g_{H}\right) q^{*} \operatorname{ch}(\bar{\xi}) \\
& +\int_{\widetilde{X}}\left(q^{*} \log \|d \pi\|^{2}\right) \widetilde{\pi}^{*} c_{1}\left([0],\|\cdot\|_{0}\right)\left[\widetilde{\mu}^{*} \operatorname{Td}(\bar{U}) \widetilde{\nu}^{*}\left\{\frac{\operatorname{Td}\left(-c_{1}(\bar{L})\right)-1}{-c_{1}(\bar{L})}\right\} q^{*} \operatorname{ch}(\bar{\xi})\right] \\
& -\int_{\widetilde{X}}\left(\widetilde{\pi}^{*} \log \left\|\mathbf{s}_{0}\right\|_{0}^{2}\right) \widetilde{\nu}^{*} c_{1}(\bar{L})\left[\widetilde{\mu}^{*} \operatorname{Td}(\bar{U}) \widetilde{\nu}^{*}\left\{\frac{\operatorname{Td}\left(-c_{1}(\bar{L})-1\right.}{-c_{1}(\bar{L})}\right\} q^{*} \operatorname{ch}(\bar{\xi})\right]+C_{1} .
\end{aligned}
$$

This completes the proof of Theorem 5.2.

Lemma 5.3. Let $\mathcal{E}: 0 \longrightarrow E^{\prime} \longrightarrow E \longrightarrow E^{\prime \prime} \longrightarrow 0$ be an exact sequence of holomorphic vector bundles over a complex manifold $Y$. Let $h^{\prime}$ and $h$ be Hermitian metrics on $E^{\prime}$ and $E$, respectively. Let $h^{\prime \prime}$ and $g^{\prime \prime}$ be Hermitian metrics on $E^{\prime \prime}$. Then

$$
\widetilde{\operatorname{Td}}\left(\mathcal{E} ; h^{\prime}, h, h^{\prime \prime}\right)-\widetilde{\mathrm{Td}}\left(\mathcal{E} ; h^{\prime}, h, g^{\prime \prime}\right)=\operatorname{Td}\left(E^{\prime}, h^{\prime}\right) \widetilde{\mathrm{Td}}\left(E^{\prime \prime} ; h^{\prime \prime}, g^{\prime \prime}\right) .
$$

Proof. Setting $\bar{L}_{1}=\left(\mathcal{E}, h^{\prime}, h, h^{\prime \prime}\right), \bar{L}_{2}=\left(\mathcal{E}, h^{\prime}, h, g^{\prime \prime}\right), \bar{L}_{3}=0$ in [10, I, Prop. 1.3.4], we get

$$
\widetilde{\operatorname{Td}}\left(\mathcal{E} ; h^{\prime}, h, h^{\prime \prime}\right)-\widetilde{\operatorname{Td}}\left(\mathcal{E} ; h^{\prime}, h, g^{\prime \prime}\right)=\widetilde{\operatorname{Td}}\left(E^{\prime} \oplus E^{\prime \prime} ; h^{\prime} \oplus h^{\prime \prime}, h^{\prime} \oplus g^{\prime \prime}\right) .
$$

Since $\widetilde{\operatorname{Td}}\left(E^{\prime} \oplus E^{\prime \prime} ; h^{\prime} \oplus h^{\prime \prime}, h^{\prime} \oplus g^{\prime \prime}\right)=\operatorname{Td}\left(E^{\prime}, h^{\prime}\right) \widetilde{\operatorname{Td}}\left(E^{\prime \prime} ; h^{\prime \prime}, g^{\prime \prime}\right)$ by [10, I, Prop. 1.3.2], we get the result. 


\section{The divergent term and the constant term}

Let $\alpha$ be a nowhere vanishing holomorphic section of $\lambda\left([\Gamma]^{-1} \otimes p_{1}^{*} \xi\right)^{-1} \otimes \lambda\left(p_{1}^{*} \xi\right)$ defined on $\mathcal{U}$.

Theorem 6.1. Let $\sigma$ be a nowhere vanishing holomorphic section of $\lambda(\xi)$ defined on $\mathcal{U}$. Then

$$
\log \|\sigma\|_{Q, \lambda(\xi)}^{2} \equiv_{\mathcal{B}}\left(\int_{E_{0}} \widetilde{\mu}^{*}\left\{\operatorname{Td}(U) \frac{\operatorname{Td}(H)-1}{c_{1}(H)}\right\} q^{*} \operatorname{ch}(\xi)\right) \log |t|^{2} .
$$

Proof. There exists a nowhere vanishing holomorphic function $f(t)$ on $\mathcal{U}$ such that

$$
\sigma(t)=f(t) \sigma_{K M}(t) \otimes \alpha(t) .
$$

Since $\log |f(t)|^{2}$ and $\log \|\alpha\|_{Q, \lambda\left([\Gamma]^{-1} \otimes p_{1}^{*} \xi\right)^{-1} \otimes \lambda\left(p_{1}^{*} \xi\right)}^{2}$ are $C^{\infty}$ functions on $\mathcal{U}$, we deduce from Theorem 5.1 that

$$
\begin{aligned}
\log \|\sigma(t)\|_{Q, \lambda(\xi)}^{2} & =\log |f(t)|^{2}+\log \left\|\sigma_{K M}(t)\right\|_{Q, \lambda}^{2}+\log \|\alpha(t)\|_{Q, \lambda\left([\Gamma]^{-1} \otimes p_{1}^{*} \xi\right)^{-1} \otimes \lambda\left(p_{1}^{*} \xi\right)}^{2} \\
& \equiv_{\mathcal{B}}\left(\int_{E_{0}} \widetilde{\mu}^{*}\left\{\operatorname{Td}(U) \frac{\operatorname{Td}(H)-1}{c_{1}(H)}\right\} q^{*} \operatorname{ch}(\xi)\right) \log |t|^{2} .
\end{aligned}
$$

This completes the proof of Theorem 6.1.

Theorem 6.2. The following identity holds:

$$
\begin{aligned}
\lim _{t \rightarrow 0} & {\left[\log \left\|\sigma_{K M} \otimes \alpha\right\|_{Q, \lambda(\xi)}^{2}(t)-\left(\int_{E_{0}} \widetilde{\mu}^{*}\left\{\operatorname{Td}(U) \frac{\operatorname{Td}(H)-1}{c_{1}(H)}\right\} q^{*} \operatorname{ch}(\xi)\right) \log \left\|\mathbf{s}_{0}(t)\right\|_{0}^{2}\right] } \\
= & \log \|\alpha(0)\|_{Q}^{2}-\left.\int_{X \times\{0\}} \frac{\operatorname{Td}\left(T X, g_{X}\right) \operatorname{ch}(\bar{\xi})}{\operatorname{Td}\left([\Gamma], h_{[\Gamma]}\right)} \log \left\|s_{\Gamma}\right\|^{2}\right|_{X \times\{0\}} \\
& -\int_{\widetilde{X}_{0}} \widetilde{\mu}^{*} \widetilde{T d}\left(\mathcal{S}^{\vee} ; g_{U},\left(\Pi^{\vee}\right)^{*} g_{X}, g_{H}\right) q^{*} \operatorname{ch}(\bar{\xi}) \\
& +\int_{\widetilde{X}}\left(q^{*} \log \|d \pi\|^{2}\right) \widetilde{\pi}^{*} c_{1}\left([0],\|\cdot\|_{0}\right)\left[\widetilde{\mu}^{*} \operatorname{Td}(\bar{U}) \widetilde{\nu}^{*}\left\{\frac{\operatorname{Td}\left(-c_{1}(\bar{L})\right)-1}{-c_{1}(\bar{L})}\right\} q^{*} \operatorname{ch}(\bar{\xi})\right] \\
& -\int_{\widetilde{X}}\left(\pi^{*} \log \left\|\mathbf{s}_{0}\right\|_{0}^{2}\right) \widetilde{\nu}^{*} c_{1}(\bar{L})\left[\widetilde{\mu}^{*} \operatorname{Td}(\bar{U}) \widetilde{\nu}^{*}\left\{\frac{\operatorname{Td}\left(-c_{1}(\bar{L})\right)-1}{-c_{1}(\bar{L})}\right\} q^{*} \operatorname{ch}(\bar{\xi})\right] \\
& -\int_{X} \operatorname{Td}(T X) \operatorname{R}(T X) \operatorname{ch}(\xi)+\int_{Z} \operatorname{Td}(T Z) \operatorname{R}(T Z) \operatorname{ch}\left(\left.\xi\right|_{Z}\right) .
\end{aligned}
$$

Proof. Since

$$
\log \left\|\sigma_{K M} \otimes \alpha\right\|_{Q, \lambda(\xi)}^{2}=\log \left\|\sigma_{K M}\right\|_{Q, \lambda}^{2}+\log \|\alpha\|_{Q, \lambda\left([\Gamma]^{-1} \otimes p_{1}^{*} \xi\right)^{-1} \otimes \lambda\left(p_{1}^{*} \xi\right)}^{2},
$$

the result follows from Theorem 5.2.

\section{Critical points defined by a quadric polynomial of rank 2}

In this section, we assume that for every $x \in \Sigma_{\pi} \cap X_{0}$, there exists a system of coordinates $\left(z_{0}, \ldots, z_{n}\right)$ centered at $x$ such that

$$
\pi(z)=z_{0} z_{1}
$$

Hence $\Sigma_{\pi} \subset X$ is a complex submanifold of codimension 2 defined locally by the equation $z_{0}=z_{1}=0$. Let $N_{\Sigma_{\pi} / X}$ be the normal bundle of $\Sigma_{\pi}$ in $X$. In [3, Def. 5.1, 
Prop. 5.2], Bismut introduced the additive genus $\mathrm{E}(\cdot)$ associated with the generating function

$$
\mathrm{E}(x):=\frac{\operatorname{Td}(x) \operatorname{Td}(-x)}{2 x}\left(\frac{\operatorname{Td}^{-1}(x)-1}{x}-\frac{\operatorname{Td}^{-1}(-x)-1}{-x}\right),
$$

where $\operatorname{Td}^{-1}(x):=\left(1-e^{-x}\right) / x$.

The following result was proved by Bismut [3, Th. 5.9].

Theorem 7.1. The following equation of functions on $\mathcal{U}^{\circ}$ holds:

$$
\log \|\sigma(t)\|_{\lambda(\xi), Q}^{2} \equiv_{\mathcal{B}} \frac{1}{2}\left(\int_{\Sigma_{\pi} \cap X_{0}}-\operatorname{Td}\left(T \Sigma_{\pi}\right) \mathrm{E}\left(N_{\Sigma_{\pi} / X}\right) \operatorname{ch}(\xi)\right) \log |t|^{2} .
$$

Remark 7.2. As mentioned before, the dual of our $\lambda(\xi)$ was defined as $\lambda(\xi)$ in $[3$, Th. 5.9], which explains the difference of the sign of the coefficient of $\log |t|^{2}$ in Theorem 7.1 with that of $[3$, Th. 5.9].

Proof. Let $q: \widetilde{X} \rightarrow X$ be the blowing-up along $\Sigma_{\pi}$ with exceptional divisor

$$
E=\mathbb{P}\left(N_{\Sigma_{\pi} / X}\right)
$$

Then $\widetilde{\nu}=\nu \circ q$ extends to a holomorphic map from $\widetilde{X}$ to $\mathbb{P}\left(\Omega_{X}^{1}\right)$.

Since the Hessian of $\pi$ is a non-degenerate symmetric bilinear form on $N_{\Sigma_{\pi} / X}$, we have $N_{\Sigma_{\pi} / X} \cong N_{\Sigma_{\pi} / X}^{*}$. Under the identification $\mathbb{P}\left(N_{\Sigma_{\pi} / X}\right)=\mathbb{P}\left(N_{\Sigma_{\pi} / X}^{*}\right)$ induced from the Hessian of $\pi, \widetilde{\nu}$ is identified with the natural inclusion $\mathbb{P}\left(N_{\Sigma_{\pi} / X}^{*}\right) \hookrightarrow$ $\mathbb{P}\left(\left.\Omega_{X}^{1}\right|_{\Sigma_{\pi}}\right)$, which yields that

$$
\left.\widetilde{\nu}^{*} L\right|_{E}=\mathcal{O}_{\mathbb{P}\left(N_{\Sigma_{\pi} / X}^{*}\right)}(-1),\left.\quad \widetilde{\mu}^{*} H\right|_{E}=\mathcal{O}_{\mathbb{P}\left(N_{\Sigma_{\pi} / X}\right)}(1) .
$$

Set $F:=\mathcal{O}_{\mathbb{P}\left(N_{\Sigma_{\pi} / X}\right)}(1)$.

By the exact sequence $\mathcal{S}^{\vee}$, we get

$$
\operatorname{Td}(U)=\frac{\operatorname{Td}\left(\left(\Pi^{\vee}\right)^{*} T X\right)}{\operatorname{Td}(H)} .
$$

Since $\Pi^{\vee} \circ \widetilde{\mu}=q$, we deduce from the exact sequence of vector bundles on $\Sigma_{\pi}$

$$
\left.0 \longrightarrow T \Sigma_{\pi} \longrightarrow T X\right|_{\Sigma_{\pi}} \longrightarrow N_{\Sigma_{\pi} / X} \longrightarrow 0
$$

the identity

$$
\left.\widetilde{\mu}^{*} \operatorname{Td}\left(\left(\Pi^{\vee}\right)^{*} T X\right)\right|_{E}=q^{*}\left\{\operatorname{Td}\left(T \Sigma_{\pi}\right) \operatorname{Td}\left(N_{\Sigma_{\pi} / X}\right)\right\} .
$$

Substituting (7.3) into (7.2), we get

$$
\left.\widetilde{\mu}^{*} \operatorname{Td}(U)\right|_{E}=\frac{q^{*}\left\{\operatorname{Td}\left(T \Sigma_{\pi}\right) \operatorname{Td}\left(N_{\Sigma_{\pi} / X}\right)\right\}}{\left.\widetilde{\mu}^{*} \operatorname{Td}(H)\right|_{E}}=\frac{q^{*}\left\{\operatorname{Td}\left(T \Sigma_{\pi}\right) \operatorname{Td}\left(N_{\Sigma_{\pi} / X}\right)\right\}}{\operatorname{Td}(F)},
$$

where we used (7.1) to get the second equality. 
Let $p_{*}$ be the integration along the fibers of the projection $p: \mathbb{P}\left(N_{\Sigma_{\pi} / X}\right) \rightarrow \Sigma_{\pi}$. Since $\left.q\right|_{E}=p$, we deduce from (7.1), (7.4) and the projection formula that

$$
\begin{aligned}
& \int_{E \cap X_{0}} \tilde{\mu}^{*}\left\{\operatorname{Td}(U) \frac{\operatorname{Td}(H)-1}{c_{1}(H)}\right\} q^{*} \operatorname{ch}(\xi) \\
& =\int_{\Sigma_{\pi} \cap X_{0}} \operatorname{Td}\left(T \Sigma_{\pi}\right) \operatorname{Td}\left(N_{\Sigma_{\pi} / X}\right) \operatorname{ch}(\xi) p_{*}\left\{\frac{1}{\operatorname{Td}(F)} \cdot \frac{\operatorname{Td}(F)-1}{c_{1}(F)}\right\} \\
& =\int_{\Sigma_{\pi} \cap X_{0}} \operatorname{Td}\left(T \Sigma_{\pi}\right) \operatorname{Td}\left(N_{\Sigma_{\pi} / X}\right) \operatorname{ch}(\xi) p_{*}\left\{\frac{1-\operatorname{Td}^{-1}(F)}{c_{1}(F)}\right\} .
\end{aligned}
$$

Since $N_{\Sigma_{\pi} / X} \cong N_{\Sigma_{\pi} / X}^{*}$, we have

$$
c_{1}\left(N_{\Sigma_{\pi} / X}\right)=0
$$

which, together with $\operatorname{rk}\left(N_{\Sigma_{\pi} / X}\right)=2$, yields that

$$
0=c_{1}(F)^{2}-p^{*} c_{1}\left(N_{\Sigma_{\pi} / X}\right) c_{1}(F)+p^{*} c_{2}\left(N_{\Sigma_{\pi} / X}\right)=c_{1}(F)^{2}+p^{*} c_{2}\left(N_{\Sigma_{\pi} / X}\right) .
$$

Since $p_{*} c_{1}(F)=1$, this implies that for $m \geq 0$

$$
p_{*} c_{1}(F)^{m}=\left\{\begin{array}{lr}
(-1)^{k} c_{2}\left(N_{\Sigma_{\pi} / X}\right)^{k} & (m=2 k+1) \\
0 & (m=2 k) .
\end{array}\right.
$$

For a formal power series $f(x)=\sum_{j=0}^{\infty} a_{j} x^{j} \in \mathbb{C}[[x]]$, set

$$
f_{-}(x):=\frac{f(x)-f(-x)}{2 x} \in \mathbb{C}[[x]] .
$$

By (7.6), we get

$$
p_{*} f\left(c_{1}(F)\right)=\sum_{k} a_{2 k+1} p_{*} c_{1}(F)^{2 k+1}=\sum_{k}(-1)^{k} a_{2 k+1} c_{2}\left(N_{\Sigma_{\pi} / X}\right)^{k} .
$$

Let $f_{-}\left(N_{\Sigma_{\pi} / X}\right)$ be the additive genus associated with $f_{-}(x) \in \mathbb{C}[[x]]$. Let $x_{1}, x_{2}$ be the Chern roots of $N_{\Sigma_{\pi} / X}$. Since $c_{1}\left(N_{\Sigma_{\pi} / X}\right)=x_{1}+x_{2}=0$, we get

$$
\begin{aligned}
f_{-}\left(N_{\Sigma_{\pi} / X}\right) & =\frac{f\left(x_{1}\right)-f\left(-x_{1}\right)}{2 x_{1}}+\frac{f\left(x_{2}\right)-f\left(-x_{2}\right)}{2 x_{2}} \\
& =\sum_{k=0}^{\infty} a_{2 k+1}\left(x_{1}^{2 k}+x_{2}^{2 k}\right) \\
& =2 \sum_{k=0}^{\infty} a_{2 k+1}\left(-x_{1} x_{2}\right)^{k} \\
& =2 \sum_{k=0}^{\infty}(-1)^{k} a_{2 k+1} c_{2}\left(N_{\Sigma_{\pi} / X}\right)^{k}=2 p_{*} f\left(c_{1}(F)\right) .
\end{aligned}
$$


Setting $f(x)=\left(\operatorname{Td}^{-1}(x)-1\right) / x$, we get

$$
\begin{aligned}
\mathrm{E}\left(N_{\Sigma_{\pi} / X}\right) & =\operatorname{Td}\left(x_{1}\right) \operatorname{Td}\left(x_{2}\right)\left\{\frac{f\left(x_{1}\right)-f\left(-x_{1}\right)}{2 x_{1}}+\frac{f\left(x_{2}\right)-f\left(-x_{2}\right)}{2 x_{2}}\right\} \\
& =2 \operatorname{Td}\left(N_{\Sigma_{\pi} / X}\right) p_{*} f\left(c_{1}(F)\right) \\
& =-2 \operatorname{Td}\left(N_{\Sigma_{\pi} / X}\right) p_{*}\left(\frac{1-\mathrm{Td}^{-1}(F)}{c_{1}(F)}\right) .
\end{aligned}
$$

By comparing (7.5) and (7.7), the desired formula follows from Theorem 6.1.

\section{Isolated critical points}

In this section, we assume that $\operatorname{Sing}\left(X_{0}\right)=\Sigma_{\pi} \cap X_{0}$ consists of isolated points. Since $\Sigma_{\pi}$ is discrete, we may identify $\mathbb{P}\left(\Omega_{X}^{1}\right)$ and $\mathbb{P}(T X)$ with the trivial projectivespace bundle on a neighborhood of $\Sigma_{\pi} \cap X_{0}$ by fixing a system of coordinates near $\Sigma_{\pi} \cap X_{0}$. Under this trivialization, we consider the Gauss maps $\nu$ and $\mu$ only on a small neighborhood of $\Sigma_{\pi} \cap X_{0}$. Then we have the following expression on a neighborhood of each $p \in \Sigma_{\pi} \cap X_{0}$ :

$$
\mu(z)=\nu(z)=\left(\frac{\partial \pi}{\partial z_{0}}(z): \cdots: \frac{\partial \pi}{\partial z_{n}}(z)\right) .
$$

For a formal power series $f(x) \in \mathbb{C}[[x]]$, let $\left.f(x)\right|_{x^{m}}$ denote the coefficient of $x^{m}$. Let $\mu(\pi, p) \in \mathbb{N}$ be the Milnor number of the isolated critical point $p$ of $\pi$. The following result was proved by the author [16, Main Th.].

Theorem 8.1. The following identity of functions on $\mathcal{U}^{\circ}$ holds:

$$
\log \|\sigma\|_{\lambda(\xi), Q}^{2} \equiv_{\mathcal{B}} \frac{(-1)^{n}}{(n+2) !} \operatorname{rk}(\xi)\left(\sum_{p \in \operatorname{Sing}\left(X_{0}\right)} \mu(\pi, p)\right) \log |t|^{2} .
$$

Proof. In Theorem 6.1, we can identify $U$ (resp. $L$ ) with the universal hyperplane bundle (resp. tautological line bundle) on $\mathbb{P}^{n}$. Then $H=L^{-1}$. Set $x:=c_{1}(H)$. Hence $\int_{\mathbb{P} n} x^{n}=1$. From the exact sequence $0 \rightarrow U \rightarrow \mathbb{C}^{n+1} \rightarrow H \rightarrow 0$, we get

$$
\operatorname{Td}(U)=\operatorname{Td}^{-1}(x)=\frac{1-e^{-x}}{x} .
$$

By substituting this and the equation $\left.q^{*} \operatorname{ch}(\xi)\right|_{E \cap \widetilde{X}_{0}}=\operatorname{rk}(\xi)$ into the formula of Theorem 6.1, we get

$$
\begin{aligned}
& \int_{E_{0}} \widetilde{\mu}^{*} \operatorname{Td}(U) \widetilde{\nu}^{*}\left\{\frac{\operatorname{Td}\left(c_{1}(H)\right)-1}{c_{1}(H)}\right\} q^{*} \operatorname{ch}(\xi) \\
& =\left.\frac{1}{\operatorname{Td}(x)} \cdot \frac{\operatorname{Td}(x)-1}{x}\right|_{x^{n}} \cdot \operatorname{rk}(\xi) \int_{E_{0}} \widetilde{\mu}^{*} c_{1}(H)^{n} \\
& =\left.\left\{\frac{1}{x}-\frac{1-e^{-x}}{x^{2}}\right\}\right|_{x^{n}} \cdot \operatorname{rk}(\xi) \int_{E_{0}} \widetilde{\mu}^{*} c_{1}(H)^{n} \\
& =\frac{(-1)^{n}}{(n+2) !} \operatorname{rk}(\xi) \int_{E_{0}} \widetilde{\mu}^{*} c_{1}(H)^{n}
\end{aligned}
$$


Since

$$
\begin{aligned}
\tilde{\pi}_{*}\left\{\tilde{\mu}^{*}\left(-c_{1}\left(L, g_{L}\right)\right)^{n} q^{*}\left(\log \|d \pi\|^{2}\right)\right\} & =\pi_{*}\left\{q^{*}\left(\log \|d \pi\|^{2}\right)\left(d d^{c} \log \|d \pi\|^{2}\right)^{n}\right\} \\
& =\sum_{p \in \operatorname{Sing}\left(X_{0}\right)} \mu(\pi, p) \log |t|^{2}+O(1)
\end{aligned}
$$

by $[16$, Th. 4.1], we get

$$
\int_{E_{0}} \widetilde{\mu}^{*} c_{1}(H)^{n}=\sum_{p \in \operatorname{Sing}\left(X_{0}\right)} \mu(\pi, p)
$$

by Corollary 4.6. The result follows from Theorem 6.1 and (8.1), (8.2).

\section{Some results on asymptotic expansion}

Let $\mathcal{A}_{\mathbb{C}}\left(\right.$ resp. $\left.\mathcal{C}_{\mathbb{C}}\right)$ be the sheaf of germs of $C^{\infty}\left(\right.$ resp. $\left.C^{0}\right)$ functions on $\mathbb{C}$. The stalk of $\mathcal{A}_{\mathbb{C}}\left(\right.$ resp. $\left.\mathcal{C}_{\mathbb{C}}\right)$ at the origin is denoted by $\mathcal{A}_{0}$ (resp. $\left.\mathcal{C}_{0}\right)$. We define

$$
\mathcal{B}_{0}:=\mathcal{A}_{0} \oplus \bigoplus_{r \in \mathbb{Q} \cap(0,1]} \bigoplus_{k=0}^{n}|t|^{2 r}(\log |t|)^{k} \cdot \mathcal{A}_{0} \subset \mathcal{C}_{0}
$$

In this section, we prove the following

Theorem 9.1. Let $\Omega \subset \mathbb{C}^{n}$ be a relatively compact domain. Let $F(z)$ be a holomorphic function on $\Omega$ with critical locus $\Sigma_{F}:=\{z \in \Omega ; d F(z)=0\}$. Let $\chi(z)$ be a $C^{\infty}(n, n)$-form with compact support in $\Omega$. Define a germ $\psi \in \mathcal{C}_{0}$ by

$$
\psi(t):=\int_{\Omega} \log |F(z)-t|^{2} \chi(z) .
$$

If $\Sigma_{F} \subset F^{-1}(0)$, then $\psi(t) \in \mathcal{B}_{0}$.

The continuity of similar integrals was studied by Bost-Gillet-Soulé [8, Sect. 1.5] in relation with the regularity of the star products of Green currents.

For the proof of Theorem 9.1, we prove some intermediary results.

Lemma 9.2. Let $\Phi$ be a $C^{\infty}(n, n)$-form with compact support in $\Omega$. Let $F_{*}(\Phi)$ be the locally integrable $(1,1)$-form on $\mathbb{C}$ defined as the integration of $\Phi$ along the fibers of $F: \Omega \rightarrow \mathbb{C}$. If $\Sigma_{F} \subset F^{-1}(0)$, then there exists a germ $A(t) \in \mathcal{B}_{0}$ such that

$$
F_{*}(\Phi)(t)=A(t) \frac{d t \wedge d \bar{t}}{|t|^{2}}, \quad A(0)=0
$$

near $0 \in \mathbb{C}$.

Proof. By Hironaka, there exists a proper holomorphic modification $\varpi: \widetilde{\Omega} \rightarrow \Omega$ such that

(i) $\varpi: \widetilde{\Omega} \backslash \varpi^{-1}\left(\Sigma_{F}\right) \rightarrow \Omega \backslash \Sigma_{F}$ is an isomorphism;

(ii) $(F \circ \varpi)^{-1}\left(\Sigma_{F}\right)$ is a normal crossing divisor of $\widetilde{\Omega}$.

Set $\widetilde{F}:=F \circ \varpi$. For any $z \in F^{-1}(0)$, there exist a system of coordinates $\left(U,\left(w_{1}, \ldots, w_{n}\right)\right)$ and integers $k_{1}, \ldots, k_{l} \geq 1, l \leq n$, such that $\widetilde{F}(w)=w_{1}^{k_{1}} \cdots w_{l}^{k_{l}}$. Define a holomorphic $(n-1)$-form on $U$ by

$$
\tau:=\frac{1}{l} \sum_{i=1}^{l} \frac{1}{k_{i}}(-1)^{i-1} w_{i} d w_{1} \wedge \cdots \wedge d w_{i-1} \wedge d w_{i+1} \wedge \cdots \wedge d w_{n} .
$$


Let $\varrho_{U}$ be a $C^{\infty}$ function with compact supported in $U$. Since $\varpi^{*} \Phi$ is a $C^{\infty}$ $(n, n)$-form on $\widetilde{\Omega}$, there exists $h(w) \in C_{0}^{\infty}(U)$ such that

$$
\varrho_{U} \varpi^{*} \Phi=h(w) d w_{1} \wedge \cdots \wedge d w_{n} \wedge d \bar{w}_{1} \wedge \cdots \wedge d \bar{w}_{n} .
$$

We define a germ $B(t) \in \mathcal{C}_{0}$ by

$$
B(t):=\int_{\widetilde{F}^{-1}(t) \cap U} h(w) \tau \wedge \bar{\tau} .
$$

Then $B(t) \in \mathcal{B}_{0}$ by $[1$, p.166, Th. 4 bis $]$. Since

$$
\widetilde{F}^{*}\left(\frac{d t}{t}\right) \wedge \tau=d w_{1} \wedge \cdots \wedge d w_{n}
$$

we get by the projection formula

$$
\begin{aligned}
\widetilde{F}_{*}\left(\varrho_{U} \varpi^{*} \Phi\right)(t) & =\widetilde{F}_{*}\left(h(w) d w_{1} \wedge \cdots \wedge d w_{n} \wedge d \bar{w}_{1} \wedge \cdots \wedge d \bar{w}_{n}\right)(t) \\
& =\frac{d t \wedge d \bar{t}}{|t|^{2}} \widetilde{F}_{*}(h(w) \tau \wedge \bar{\tau})=B(t) \frac{d t \wedge d \bar{t}}{|t|^{2}} .
\end{aligned}
$$

For an $\epsilon>0$ small enough, set $\Delta(\epsilon):=\{t \in \mathbb{C} ;|t|<\epsilon\}$. Since

$$
\left|\int_{\Delta(\epsilon)} \widetilde{F}_{*}\left(\varrho_{U} \varpi^{*} \Phi\right)\right|=\left|\int_{\widetilde{F}^{-1}(\Delta(\epsilon))} \varrho_{U} \varpi^{*} \Phi\right|<\infty,
$$

the (1,1)-form $B(t) d t \wedge d \bar{t} /|t|^{2}$ is locally integrable near the origin. Hence $B(0)=0$.

Let $\left\{U_{\beta}\right\}_{\beta \in B}$ be a locally finite open covering of $\widetilde{\Omega}$ and let $\left\{\varrho_{\beta}\right\}_{\beta \in B}$ be a partition of unity subject to $\left\{U_{\beta}\right\}_{\beta \in B}$. By (9.1), there exists $B_{\beta}(t) \in \mathcal{B}_{0}$ for each $\beta \in B$ such that

$$
\widetilde{F}_{*}\left(\varrho_{\beta} \varpi^{*}(\Phi)\right)=B_{\beta}(t) \frac{d t \wedge d \bar{t}}{|t|^{2}}, \quad B_{\beta}(0)=0 .
$$

There exist finitely many $\beta \in B$ with $B_{\beta}(t) \neq 0$ by the compactness of the support of $\varpi^{*} \Phi$. Since

$$
F_{*}(\Phi)=\sum_{\beta \in B} \widetilde{F}_{*}\left(\varrho_{\beta} \varpi^{*} \Phi\right)=\left(\sum_{\beta \in B} B_{\beta}(t)\right) \frac{d t \wedge d \bar{t}}{|t|^{2}},
$$

we get $A(t)=\sum_{\beta \in B} B_{\beta}(t) \in \mathcal{B}_{0}$ and $A(0)=0$.

We regard $\Omega$ as a domain in $\left(\mathbb{P}^{1}\right)^{n}$. Hence $\chi$ is a $C^{\infty}(n, n)$-form on $\left(\mathbb{P}^{1}\right)^{n}$. Let $z=\left(z_{1}, \ldots, z_{n}\right)$ be the inhomogeneous coordinates of $\left(\mathbb{P}^{1}\right)^{n}$. For $1 \leq i \leq n$, set

$$
\omega_{i}:=\frac{\sqrt{-1} d z_{i} \wedge d \bar{z}_{i}}{2 \pi\left(1+\left|z_{i}\right|^{2}\right)^{2}}
$$

Lemma 9.3. Assume that $F(z)=z_{1}^{\nu_{1}} \cdots z_{n}^{\nu_{n}}, \nu_{1}, \ldots, \nu_{n} \geq 0$ and set

$$
\alpha:=\int_{\left(\mathbb{P}^{1}\right)^{n}} \chi(z) .
$$

Then there exists $\eta(t) \in \mathcal{B}_{0}$ such that

$$
\psi(t)=\alpha \int_{\left(\mathbb{P}^{1}\right)^{n}} \log \left|z_{1}^{\nu_{1}} \cdots z_{n}^{\nu_{n}}-t\right|^{2} \omega_{1} \wedge \cdots \wedge \omega_{n}+\eta(t) .
$$


Proof. Let $\left(\left(\zeta_{1}: \xi_{1}\right), \ldots,\left(\zeta_{n}: \xi_{n}\right)\right)$ be the homogeneous coordinates of $\left(\mathbb{P}^{1}\right)^{n}$ such that $z_{i}=\zeta_{i} / \xi_{i}$. For $t \in \mathbb{C}$, set

$$
\begin{gathered}
Y_{t}:=\left\{\left(\left(\zeta_{1}: \xi_{1}\right), \ldots,\left(\zeta_{n}: \xi_{n}\right)\right) \in\left(\mathbb{P}^{1}\right)^{n} ; \zeta_{1}^{\nu_{1}} \cdots \zeta_{n}^{\nu_{n}}-t \xi_{1}^{\nu_{1}} \cdots \xi_{n}^{\nu_{n}}=0\right\}, \\
D:=\left\{\left(\left(\zeta_{1}: \xi_{1}\right), \ldots,\left(\zeta_{n}: \xi_{n}\right)\right) \in\left(\mathbb{P}^{1}\right)^{n} ; \xi_{1}^{\nu_{1}} \cdots \xi_{n}^{\nu_{n}}=0\right\} .
\end{gathered}
$$

Since

$$
z_{1}^{\nu_{1}} \cdots z_{n}^{\nu_{n}}-t=\frac{\zeta_{1}^{\nu_{1}} \cdots \zeta_{n}^{\nu_{n}}-t \xi_{1}^{\nu_{1}} \cdots \xi_{n}^{\nu_{n}}}{\xi_{1}^{\nu_{1}} \cdots \xi_{n}^{\nu_{n}}}
$$

we get the following equation of currents on $\left(\mathbb{P}^{1}\right)^{n}$ by the Poincaré-Lelong formula:

$$
d d^{c} \log \left|z_{1}^{\nu_{1}} \cdots z_{n}^{\nu_{n}}-t\right|^{2}=\delta_{Y_{t}}-\delta_{D} .
$$

Since $\chi(z)$ is cohomologous to $\alpha \omega_{1} \wedge \cdots \wedge \omega_{n}$, there exists a $C^{\infty}(n-1, n-1)$-form $\gamma$ on $\left(\mathbb{P}^{1}\right)^{n}$ by the $d d^{c}$-Poincaré lemma, such that

$$
\chi(z)-\alpha \omega_{1} \wedge \cdots \wedge \omega_{n}=d d^{c} \gamma .
$$

Hence we get by $(9.3)$

$$
\begin{aligned}
\psi(t) & =\alpha \int_{\left(\mathbb{P}^{1}\right)^{n}} \log \left|z_{1}^{\nu_{1}} \cdots z_{n}^{\nu_{n}}-t\right|^{2} \omega_{1} \wedge \cdots \wedge \omega_{n}+\int_{\left(\mathbb{P}^{1}\right)^{n}} \log \left|z_{1}^{\nu_{1}} \cdots z_{n}^{\nu_{n}}-t\right|^{2} d d^{c} \gamma \\
& =\alpha \int_{\left(\mathbb{P}^{1}\right)^{n}} \log \left|z_{1}^{\nu_{1}} \cdots z_{n}^{\nu_{n}}-t\right|^{2} \omega_{1} \wedge \cdots \wedge \omega_{n}+\int_{\left(\mathbb{P}^{1}\right)^{n}} d d^{c}\left(\log \left|z_{1}^{\nu_{1}} \cdots z_{n}^{\nu_{n}}-t\right|^{2}\right) \wedge \gamma \\
& =\alpha \int_{\left(\mathbb{P}^{1}\right)^{n}} \log \left|z_{1}^{\nu_{1}} \cdots z_{n}^{\nu_{n}}-t\right|^{2} \omega_{1} \wedge \cdots \wedge \omega_{n}+\int_{Y_{t}} \gamma-\int_{D} \gamma .
\end{aligned}
$$

For $t \in \mathbb{C}$, set

$$
\eta(t):=\int_{Y_{t}} \gamma-\int_{D} \gamma
$$

Define a divisor of $\left(\mathbb{P}^{1}\right)^{n} \times \mathbb{C}$ by

$$
Y:=\left\{\left(\left(\zeta_{1}: \xi_{1}\right), \ldots,\left(\zeta_{n}: \xi_{n}\right), t\right) \in\left(\mathbb{P}^{1}\right)^{n} \times \mathbb{C} ; \zeta_{1}^{\nu_{1}} \cdots \zeta_{n}^{\nu_{n}}-t \xi_{1}^{\nu_{1}} \cdots \xi_{n}^{\nu_{n}}=0\right\} .
$$

Let $\operatorname{pr}_{1}:\left(\mathbb{P}^{1}\right)^{n} \times \mathbb{C} \rightarrow\left(\mathbb{P}^{1}\right)^{n}$ and $\operatorname{pr}_{2}:\left(\mathbb{P}^{1}\right)^{n} \times \mathbb{C} \rightarrow \mathbb{C}$ be the projections. Then $Y_{t}=Y \cap \operatorname{pr}_{2}^{-1}(t)$. Let $P: \widetilde{Y} \rightarrow Y$ be the resolution of the singularities of $Y$. Then $\left.\mathrm{pr}_{2}\right|_{Y} \circ P$ is a proper holomorphic function on the complex manifold $\widetilde{Y}$. Since $P^{*}\left(\mathrm{pr}_{1}\right)^{*} \gamma$ is a $C^{\infty}(n-1, n-1)$-form on $\tilde{Y}$, we get

$$
\eta(t)=\int_{\left(\operatorname{pr}_{2} \mid Y \circ P\right)^{-1}(t)} P^{*}\left(\operatorname{pr}_{1}\right)^{*} \gamma-\int_{D} \gamma \in \mathcal{B}_{0}
$$

by $[1$, Th. 4 bis]. The result follows from (9.4), (9.5), (9.6).

Define a germ $f \in \mathcal{C}_{0}$ by

$$
f(t):=\int_{\left(\mathbb{P}^{1}\right)^{n}} \log \left|z_{1}^{\nu_{1}} \cdots z_{n}^{\nu_{n}}-t\right|^{2} \omega_{1} \wedge \cdots \wedge \omega_{n} .
$$

Lemma 9.4. There exists a germ $g(t) \in \mathcal{B}_{0}$ such that

$$
d d^{c} f(t)=\frac{\sqrt{-1}}{4 \pi} g(t) \frac{d t \wedge d \bar{t}}{|t|^{2}}, \quad g(0)=0 .
$$


Proof. We keep the notation in the proof of Lemma 9.3. Since the assertion is obvious when $\nu_{1}=\cdots=\nu_{n}=0$, we assume that $\nu_{i}>0$ for some $i$. Since $z_{1}^{\nu_{1}} \cdots z_{n}^{\nu_{n}}-t$ is a meromorphic function on $\left(\mathbb{P}^{1}\right)^{n} \times \mathbb{C}$, we deduce from (9.2) and the Poincaré-Lelong formula the following equation of currents on $\left(\mathbb{P}^{1}\right)^{n} \times \mathbb{C}$ :

$$
d d^{c} \log \left|z_{1}^{\nu_{1}} \cdots z_{n}^{\nu_{n}}-t\right|^{2}=\delta_{Y}-\delta_{D \times \mathbb{C}}=\delta_{Y}-\delta_{\left(\mathrm{pr}_{1}\right)^{*} D}
$$

Since

$$
f=\left(\operatorname{pr}_{2}\right) *\left\{\log \left|z_{1}^{\nu_{1}} \cdots z_{n}^{\nu_{n}}-t\right|^{2}\left(\operatorname{pr}_{1}\right)^{*}\left(\omega_{1} \wedge \cdots \wedge \omega_{n}\right)\right\},
$$

we get on $\mathbb{C} \backslash\{0\}$

$$
\begin{aligned}
d d^{c} f & =\left(\operatorname{pr}_{2}\right)_{*}\left\{d d^{c} \log \left|z_{1}^{\nu_{1}} \cdots z_{n}^{\nu_{n}}-t\right|^{2} \wedge\left(\operatorname{pr}_{1}\right)^{*}\left(\omega_{1} \wedge \cdots \wedge \omega_{n}\right)\right\} \\
& =\left(\operatorname{pr}_{2}\right)_{*}\left\{\left(\delta_{Y}-\delta\left(\mathrm{pr}_{1}\right)^{*}\right) \wedge\left(\mathrm{pr}_{1}\right)^{*}\left(\omega_{1} \wedge \cdots \wedge \omega_{n}\right)\right\} \\
& =\left(\operatorname{pr}_{2}\right)_{*}\left\{\left.\left(\operatorname{pr}_{1}\right)^{*}\left(\omega_{1} \wedge \cdots \wedge \omega_{n}\right)\right|_{Y}\right\}-\left(\operatorname{pr}_{2}\right)_{*}\left\{\left(\operatorname{pr}_{1}\right)^{*}\left(\left.\omega_{1} \wedge \cdots \wedge \omega_{n}\right|_{D}\right)\right\} \\
& =\left(\left.\operatorname{pr}_{2}\right|_{Y}\right)_{*}\left\{\left.\left(\operatorname{pr}_{1}\right)^{*}\left(\omega_{1} \wedge \cdots \wedge \omega_{n}\right)\right|_{Y}\right\} \\
& =\left(\left.\operatorname{pr}_{2}\right|_{Y} \circ P\right)_{*}\left\{P^{*}\left(\operatorname{pr}_{1}\right)^{*}\left(\omega_{1} \wedge \cdots \wedge \omega_{n}\right)\right\},
\end{aligned}
$$

where the first equality follows from the commutativity $d d^{c}\left(\mathrm{pr}_{2}\right)_{*}=\left(\mathrm{pr}_{2}\right)_{*} d d^{c}$, the second equality follows from (9.7), and the fourth equality follows from the trivial identity $\left.\omega_{1} \wedge \cdots \wedge \omega_{n}\right|_{D}=0$. Since $P^{*}\left(\operatorname{pr}_{1}\right)^{*}\left(\omega_{1} \wedge \cdots \wedge \omega_{n}\right)$ is a $C^{\infty}(n, n)$-form on $\widetilde{Y}$ and since $\left.\operatorname{pr}_{2}\right|_{Y} \circ P: \widetilde{Y} \rightarrow \mathbb{C}$ is a proper holomorphic map, the assertion follows from (9.8) and Lemma 9.2.

Lemma 9.5. The germ $f(t)$ is $S^{1}$-invariant, i.e., $f(t)=f(|t|)$.

Proof. Without loss of generality, we may assume that $\nu_{n}>0$. Since

$$
\int_{\mathbb{P}^{1}} \log \left|A z_{n}^{\nu_{n}}+B\right|^{2} \omega_{n}=\log \left(|A|^{2 / \nu_{n}}+|B|^{2 / \nu_{n}}\right)
$$

when $(A, B) \neq(0,0)$, we get by Fubini's theorem

$$
\begin{aligned}
f(t) & =\int_{\left(\mathbb{P}^{1}\right)^{n}} \log \left|z_{1}^{\nu_{1}} \cdots z_{n}^{\nu_{n}}-t\right|^{2} \omega_{1} \wedge \cdots \wedge \omega_{n} \\
& =\int_{\left(\mathbb{P}^{1}\right)^{n-1}}\left(\int_{\mathbb{P}^{1}} \log \left|z_{1}^{\nu_{1}} \cdots z_{n}^{\nu_{n}}-t\right|^{2} \omega_{n}\right) \omega_{1} \wedge \cdots \wedge \omega_{n-1} \\
& =\int_{\left(\mathbb{P}^{1}\right)^{n-1}} \log \left(\left|z_{1}^{\nu_{1}} \cdots z_{n-1}^{\nu_{n-1}}\right|^{2 / \nu_{n}}+|t|^{2 / \nu_{n}}\right) \omega_{1} \wedge \cdots \wedge \omega_{n-1} .
\end{aligned}
$$

The assertion follows from (9.9).

Let $(r, \theta)$ be the polar coordinates of $\mathbb{C}$. Hence $t=r e^{i \theta}$.

Lemma 9.6. Let $\lambda(t) \in C^{\infty}\left(\Delta^{*}\right)$. Assume that $\lambda(t)$ is $S^{1}$-invariant, i.e., $\lambda(t)=$ $\lambda(r)$. If $r \partial_{r} \lambda(t) \in \mathcal{B}_{0}$, then $\lambda(t) \in \mathcal{B}_{0}$.

Proof. By the definition of $\mathcal{B}_{0}$, there exist a finite set $A \subset \mathbb{Q} \cap(0,1]$ and germs $\mu_{\alpha, k}(t) \in \mathcal{A}_{0}, \alpha \in A, 0 \leq k \leq n$ such that

$$
r \partial_{r} \lambda(r)=\sum_{\alpha \in A} \sum_{k=0}^{n} r^{2 \alpha}(\log r)^{k} \mu_{\alpha, k}(t) .
$$


We may assume that $\mu_{\alpha, k}(t) \in C^{\infty}(\Delta(2 \epsilon))$ for some $\epsilon>0$. Since the left hand side of (9.10) is $S^{1}$-invariant, we may assume that $\mu_{\alpha, k}(t)=\mu_{\alpha, k}(r)$ for all $\alpha$ and $k$ after replacing $\mu_{\alpha, k}(t)$ by $\int_{0}^{2 \pi} \mu_{\alpha, k}\left(e^{i \theta} t\right) d \theta / 2 \pi$. By (9.10), we get

$$
\lambda(\epsilon)-\lambda(r)=\sum_{\alpha \in A} \sum_{k=0}^{n} \int_{r}^{\epsilon} u^{2 \alpha-1}(\log u)^{k} \mu_{\alpha, k}(u) d u .
$$

By (9.11), we see that $\lambda(t) \in \mathcal{C}_{0}$ by setting

$$
\lambda(0):=\lambda(\epsilon)-\sum_{\alpha \in A} \sum_{k=0}^{n} \int_{0}^{\epsilon} u^{2 \alpha-1}(\log u)^{k} \mu_{\alpha, k}(u) d u .
$$

Since $\lambda(t) \in \mathcal{C}_{0}$, we get by $(9.11)$

$$
\begin{aligned}
\lambda(r) & =\lambda(0)+\sum_{\alpha \in A} \sum_{k=0}^{n} \int_{0}^{r} u^{2 \alpha-1}(\log u)^{k} \mu_{\alpha, k}(u) d u \\
& =\lambda(0)+\sum_{\alpha \in A} \sum_{k=0}^{n} r^{2 \alpha} \int_{0}^{1} v^{2 \alpha-1}(\log r+\log v)^{k} \mu_{\alpha, k}(v r) d v \\
& =\lambda(0)+\sum_{\alpha \in A} \sum_{k=0}^{n} \sum_{l=0}^{k}\left(\begin{array}{l}
k \\
l
\end{array}\right) r^{2 \alpha}(\log r)^{l} \int_{0}^{1} v^{2 \alpha-1}(\log v)^{k-l} \mu_{\alpha, k}(v t) d v,
\end{aligned}
$$

which implies that $\lambda(t) \in \mathcal{B}_{0}$.

Lemma 9.7. If $F(z)=z_{1}^{\nu_{1}} \cdots z_{n}^{\nu_{n}}, \nu_{1}, \ldots, \nu_{n} \geq 0$, then $\psi(t) \in \mathcal{B}_{0}$.

Proof. By Lemma 9.3, it suffices to prove that $f \in \mathcal{B}_{0}$. Since $f(t)=f(r)$ by Lemma 9.5, we deduce from Lemma 9.4 the equation

$$
\frac{1}{2 \pi} \partial_{t} \partial_{\bar{t}} f(t)=\frac{1}{4 \pi}\left\{f^{\prime \prime}(r)+r^{-1} f^{\prime}(r)\right\}=\frac{g(t)}{4 \pi r^{2}} .
$$

Hence $g(t)$ is invariant under the rotation, i.e., $g(t)=g(r)$, and the following equation holds

$$
\left(r \partial_{r}\right)^{2} f(r)=g(r)
$$

Since $g(t) \in \mathcal{B}_{0}$, we deduce from Lemma 9.6 and (9.12) that $r \partial_{r} f(r) \in \mathcal{B}_{0}$. By Lemma 9.6 again, we get $f(t) \in \mathcal{B}_{0}$.

\section{Proof of Theorem 9.1}

We keep the notation in the proof of Lemma 9.2. There exists a system of coordinate neighborhoods $\left\{\left(U_{\beta}, w_{\beta}=\left(w_{1, \beta}, \ldots, w_{n, \beta}\right)\right)\right\}_{\beta \in B}$ of $\widetilde{\Omega}$ and integers $k_{1, \beta}, \ldots, k_{n, \beta} \geq$ 0 for each $\beta \in B$ such that $\left.\widetilde{F}\right|_{U_{\beta}}\left(w_{\beta}\right)=w_{1, \beta}^{k_{1, \beta}} \cdots w_{n, \beta}^{k_{n, \beta}}$. Without loss of generality, we may assume that the covering $\left\{U_{\beta}\right\}_{\beta \in B}$ of $\widetilde{\Omega}$ is locally finite. Let $\left\{\varrho_{\beta}\right\}_{\beta \in B}$ be a partition of unity subject to the covering $\left\{U_{\beta}\right\}_{\beta \in B}$. Then $\chi_{\beta}:=\varrho_{\beta} \varpi^{*} \chi$ is a $C^{\infty}$ $(n, n)$-form with compact support in $U_{\beta}$. Since $\varpi^{*} \chi$ has a compact support in $\widetilde{\Omega}$, $\chi_{\beta}=0$ except finitely many $\beta \in B$. By Lemma 9.7 ,

$$
\psi_{\beta}(t):=\int_{U_{\beta}} \log \left|w_{1, \beta}^{k_{1, \beta}} \cdots w_{n, \beta}^{k_{n, \beta}}-t\right|^{2} \chi_{\beta}\left(w_{\beta}\right) \in \mathcal{B}_{0}
$$


Since

$\psi(t)=\int_{\widetilde{\Omega}} \varpi^{*} \log |F-t|^{2} \varpi^{*} \chi=\sum_{\beta \in B} \int_{U_{\beta}} \log |\widetilde{F}|_{U_{\beta}}\left(w_{\beta}\right)-\left.t\right|^{2} \varrho_{\beta} \varpi^{*} \chi=\sum_{\beta \in B} \psi_{\beta}(t)$,

we get $\psi(t) \in \mathcal{B}_{0}$ by (9.13). This completes the proof of Theorem 9.1.

\section{REFERENCES}

[1] Barlet, D. Développement asymptotique des fonctions obtenues par intégration sur les fibres, Invent. Math. 68 (1982), 129-174

[2] Bismut, J.-M. Superconnection currents and complex immersions, Invent. Math. 99 (1990), $59-113$

[3] Quillen metrics and singular fibers in arbitrary relative dimension, Jour. Algebr. Geom. 6 (1997), 19-149

[4] Bismut, J.-M., Bost, J.-B. Fibrés déterminants, métriques de Quillen et dégénérescence des courbes, Acta Math 165 (1990) 1-103

[5] Bismut, J.-M., Gillet, H., Soulé, C. Analytic torsion and holomorphic determinant bundles I,II,III, Commun. Math. Phys. 115 (1988), 49-78, 79-126, 301-351

[6] Complex immersions and Arakelov geometry, (P. Cartier et al., eds.), The Grothendieck Festschrift, Birkhäuser, Boston (1990) 249-331

[7] Bismut, J.-M., Lebeau, G. Complex immersions and Quillen metrics, Publ. Math. IHES 74 (1991), 1-297

[8] Bost, J.-B., Gillet, H., Soulé, C. Hights of projective varieties and positive Green forms, Jour. Amer. Math. Soc. 7 (1994), 903-1027

[9] Fang, H., Lu, Z., Yoshikawa, K.-I. Analytic torsion for Calabi-Yau threefolds, E-print, arXiv: math.DG/0601411 (2006)

[10] Gillet, H., Soulé, C. Characteristic classes for algebraic vector bundles with hermitian metric, I,II Ann. of Math. 131 (1990), 163-238

[11] Arithmetic intersection theory, Publ. Math. IHES 72 (1990), 93-174

[12] Knudsen, F.F., Mumford, D. The projectivity of the moduli space of stable curves, I., Math. Scand. 39 (1976), 19-55

[13] Noguchi, J., Ochiai, T. Geometric Function Theory in Several Complex Variables, Amer. Math. Soc., (1990)

[14] Quillen, D. Determinants of Cauchy-Riemann operators over a Riemann surface, Funct. Anal. Appl. 14 (1985), 31-34

[15] Soulé, C. et al. Lectures on Arakelov Geometry, Cambridge University Press, Cambridge (1992)

[16] Yoshikawa, K.-I. Smoothing of isolated hypersurface singularities and Quillen metrics, Asian J. Math. 2 (1998), 325-344

[17] K3 surfaces with involution, equivariant analytic torsion, and automorphic forms on the moduli space, Invent. Math. 156 (2004), 53-117

Graduate School of Mathematical Sciences, University of Tokyo, 3-8-1 Komaba, TOKYO 153-8914, JAPAN

E-mail address: yosikawa@ms.u-tokyo.ac.jp 